\title{
Stellar probes of dark sector-photon interactions
}

\author{
Xiaoyong Chu ${ }^{*}$, Jui-Lin Kuo๑, ${ }^{\dagger}$ Josef Pradler, ${ }^{\star}$ and Lukas Semmelrock $\odot^{\S}$ \\ Institute of High Energy Physics, Austrian Academy of Sciences, \\ Nikolsdorfergasse 18, 1050 Vienna, Austria
}

(Received 12 August 2019; published 3 October 2019)

\begin{abstract}
Electromagnetically neutral dark sector particles may directly couple to the photon through higher dimensional effective operators. Considering the electric and magnetic dipole moment, anapole moment, and charge radius interactions, we derive constraints from the stellar energy loss in the Sun, horizontal branch and red giant stars, as well as from cooling of the proto-neutron star of SN1987A. We provide the exact formula for in-medium photon-mediated pair production to leading order in the dark coupling and compute the energy loss rates explicitly for the most important processes, including a careful discussion on resonances and potential double counting between the processes. Stringent limits for dark states with masses below $3 \mathrm{keV}(40 \mathrm{MeV}$ ) arise from red giant stars (SN1987A), implying an effective lower massscale of approximately $10^{9} \mathrm{GeV}\left(10^{7} \mathrm{GeV}\right)$ for mass-dimension five and $100 \mathrm{GeV}(2.5 \mathrm{TeV})$ for massdimension six operators as long as dark states stream freely; for the proto-neutron star, the trapping of dark states is also evaluated. Together with direct limits previously derived by us in Chu et al. (2018), this provides the first comprehensive overview of the viability of effective electromagnetic dark-state interactions below the GeV mass-scale.
\end{abstract}

DOI: $10.1103 /$ PhysRevD.100.083002

\section{INTRODUCTION}

The prospect that new physics might be hiding under our noses in the form of light dark states that have been in kinematic reach for decades is most intriguing if not seemingly preposterous. In fact, cases exist where new interactions are of comparable strength to the ones encountered in the Standard Model (SM), while being compatible with all to-date searches. The direct test of such physics, i.e., new particles and interactions below the $\mathrm{GeV}$-scale has become a major field in recent years $[1,2]$ and provides a complementary direction to the beyond-SM searches at the energy frontier.

Whereas the GeV-mass scale might comprise somewhat of a "blind-spot" that allows for the existence of new physics with appreciable interactions to the SM, once the mass enters the keV-regime the landscape changes fundamentally. Astrophysical constraints on long-lived dark states that are derived from stellar cooling arguments [3] are typically so severe that the cases for laboratory

\footnotetext{
*xiaoyong.chu@oeaw.ac.at

jui-lin.kuo@oeaw.ac.at

\#josef.pradler@oeaw.ac.at

§lukas.semmelrock@oeaw.ac.at
}

Published by the American Physical Society under the terms of the Creative Commons Attribution 4.0 International license. Further distribution of this work must maintain attribution to the author(s) and the published article's title, journal citation, and DOI. Funded by SCOAP ${ }^{3}$. detection drastically diminish. Of course, the observable signatures of dark states depend on the nature of the coupling to the SM. For example, a new force can be mediated by new scalar or vector particles. Benchmark models are then derived based on minimality of the SM extension and on the dimensionality of the interaction operator, and within this framework the interplay between cosmological and astrophysical implications and direct tests is fleshed out.

A prominent example is the vector portal, where the low-energy phenomenology is determined by the kinetic mixing strength $\epsilon$ of the "dark photon" $V$ with the SM photon [4]. If the mass of $V, m_{V}$, originates from a Higgs mechanism, implying an additional scalar particle in the vicinity of $m_{V}$, stellar cooling constraints obliterate any prospects of probing such a model below the keV-region, as limits on millicharged particles apply. However, a decoupling of stellar constraints as $\epsilon^{2} m_{V}^{2}$ [5] when $m_{V}$ arises from a Stuckelberg mechanism, opens the opportunity to explore a vast parameter region through direct, laboratory searches, in particular if $V$ is the dark matter (DM); see, e.g., [6-11,11-20].

In this work we will consider-from the low-energy effective theory point of view-an even more minimal possibility than the dark photon, namely, that the SM photon is the new physics mediator. Beyond carrying a millicharge, DM may also interact directly with the photon through a number of higher dimensional operators that encapsulate magnetic or electric dipole moment 


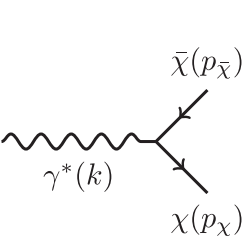

(a)

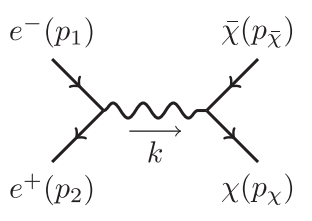

(b)

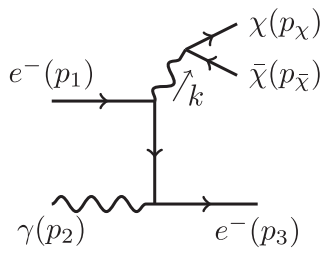

(c)

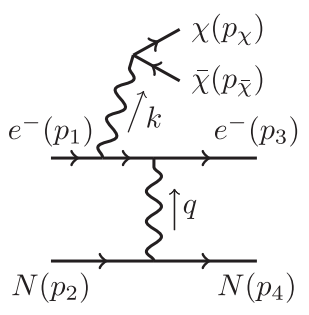

(d)

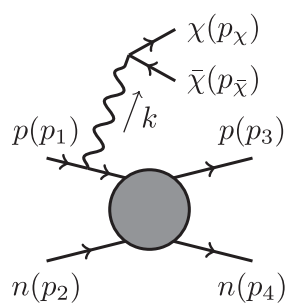

(e)

FIG. 1. Shown are the pair production processes of $\chi \bar{\chi}$ that are calculated in this paper, namely, (a) plasmon decay, (b) $e^{+} e^{-}$ annihilation, (c) $2 \rightarrow 3$ Compton scattering, (d) electron bremsstrahlung and (e) nucleon bremsstrahlung; for (c)-(d) we only show one of two relevant diagrams. The four momentum of the $\chi \bar{\chi}$-producing photon is denoted by $k$ throughout the paper.

interactions (MDM or EDM), an anapole moment (AM) or a charge radius interaction (CR). These possibilities were originally considered in [21-23] with further studies on the phenomenology found in [24-29]. Motivated by the intense efforts to search for sub-GeV dark sector states [1,2], the topic of form-factor interactions was recently revisited in detail by some of us [30].

In [30] we focused on the prospects of detecting electromagnetic (EM) form factor interactions of a dark sector Dirac particle $\chi$ with mass at or below the GeVscale. The direct production of pairs $\chi \bar{\chi}$ was constrained with data from BABAR [31], NA64 [32] and mQ [33] and future improvements in sensitivity were derived for Belle-II [34], LDMX [35] and BDX [36]. The direct sensitivity was then compared with indirect probes such as electroweak precision tests, flavor physics constraints, as well as with results from LEP and LHC. It was found that, owing to the higher dimensionality of the operators, high energy probes provide superior sensitivity. The conclusions are independent of the lifetime of $\chi$, as long as its stability is guaranteed while traversing terrestrial detectors.

In contrast, if $\chi$ is long-lived, additional constraints from cosmology, astrophysics, and direct DM searches apply, and in [30] we have considered the most important ones that are crucial in the $\mathrm{MeV}-\mathrm{GeV}$ mass bracket of $\chi$. However, once we allow the $\chi$-mass to drop into the $\mathrm{keV}$-region, additional constraints from the production of $\chi \bar{\chi}$-pairs in stars become important [3]. In this work we complement our previous results derived in [30] with astrophysical limits that apply once the dark state is stable on a macroscopic time scale, without necessarily demanding that sub-MeV $\chi$ particles make up the DM. We derive the limits from stellar cooling that arise from red giant (RG), horizontal branch (HB) stars, and the Sun, and revisit our calculation of the supernova bound, taking into account all major production channels.

Concretely, we are considering the following fundamental dark state emission processes, highlighting in brackets the stellar system(s) for which the process is most relevant,
Plasmon decay: $\quad \gamma_{\mathrm{T}, \mathrm{L}} \rightarrow \chi \bar{\chi} \quad($ all $)$,

Annihilation:

$$
e^{+} e^{-} \rightarrow \chi \bar{\chi} \quad(\mathrm{SN}),
$$

Bremsstrahlung: $\quad e^{-} N \rightarrow e^{-} N \chi \bar{\chi} \quad(\mathrm{RG}, \mathrm{HB}$, Sun $)$,

$$
N N \rightarrow N N \chi \bar{\chi} \quad(\mathrm{SN}),
$$

Compton scattering: $e^{-} \gamma_{\mathrm{T}, \mathrm{L}} \rightarrow e^{-} \chi \bar{\chi} \quad$ (all).

The respective processes are decay of in-medium longitudinal (L) and transverse (T) modes of thermal photons $\gamma_{T, L}$ which we will simply refer to as "plasmons", electronpositron annihilation, electron bremsstrahlung on protons and nuclei, nucleon-nucleon bremsstrahlung and Compton scattering with the emission of a $\chi \bar{\chi}$-pair. Exemplary respective diagrams are shown in Fig. 1.

The paper is organized as follows: in Sec. II we first set the stage by listing the effective operators that mediate $\chi$ photon interactions. Section III gives a brief account on stellar energy loss arguments. Our calculations on $\chi$ particle emission are presented in Sec. IV. The ensuing constraints are then collected in Sec. V before concluding in Sec. VI. Several Appendixes provide details on the calculations and are referenced in the main text.

\section{ELECTROMAGNETIC FORM FACTOR INTERACTIONS}

A Dirac fermion $\chi$ may have a number of interactions with the photon gauge field $A_{\mu}$ or its field strength tensor $F_{\mu \nu}$. At mass dimension-5 the interaction terms of the Lagrangian are given by

$$
\mathcal{L}_{\chi}^{\operatorname{dim}-5}=\frac{1}{2} \mu_{\chi} \bar{\chi} \sigma^{\mu \nu} \chi F_{\mu \nu}+\frac{i}{2} d_{\chi} \bar{\chi} \sigma^{\mu \nu} \gamma^{5} \chi F_{\mu \nu},
$$

where $\mu_{\chi}$ and $d_{\chi}$ are the MDM and EDM coupling which may be measured in units of the Bohr magneton, $\mu_{B} \equiv$ $e /\left(2 m_{e}\right)=1.93 \times 10^{-11} e \mathrm{~cm} ; m_{e}$ is the mass of the electron and $\sigma^{\mu \nu}=\frac{i}{2}\left[\gamma^{\mu}, \gamma^{\nu}\right]$. At mass dimension- 6 we have 


$$
\mathcal{L}_{\chi}^{\operatorname{dim}-6}=-a_{\chi} \bar{\chi} \gamma^{\mu} \gamma^{5} \chi \partial^{\nu} F_{\mu \nu}+b_{\chi} \bar{\chi} \gamma^{\mu} \chi \partial^{\nu} F_{\mu \nu},
$$

where $a_{\chi}$ and $b_{\chi}$ are the $\mathrm{AM}$ and CR coefficients. All coupling strengths in Eqs. (5) and (6) are real. At massdimension-7 the interactions involve two photons at the vertex and hence require a dedicated treatment. For this reason we restrict our study to dim-5 and dim- 6 operators.

The effective interactions in Eqs. (5) and (6) may, e.g., arise from the compositeness of $\chi$ [37-39] or perturbatively, from a UV completion that contains electrically charged states [40]. In the latter case, MDM and EDM moments are e.g., generated by loop-induced axial or vector Yukawa interactions $y_{A, V}$ of $\chi$ with additional scalars and fermions. Parametrically, one expects $\mu_{\chi} \sim Q\left|y_{A, V}\right|^{2} / M$ and $d_{\chi} \sim$ $Q \operatorname{Im}\left[y_{V} y_{A}^{*}\right] / M$ where $Q$ is the electric charge of the mediator and $M$ is some common mass-scale of these new states. In turn, the strength of AM and CR interactions may be expected as $a_{\chi}, b_{\chi} \sim Q\left|y_{A, V}\right|^{2} / M^{2}$. It should be noted, however, that these estimates may be significantly enhanced by the lightness and/or mass-degeneracy of the spectrum of states [41]; a systematic study on EDMs induced by $C P$ violation from light dark sectors was recently performed in [42]. In what follows, we treat the interactions (5) and (6) independent of their embedding.

For the Feynman-diagrammatic computation, one assembles the interactions into the matrix element of the effective EM current of $\chi$,

$$
\left\langle\chi\left(p_{f}\right)\left|J_{\chi}^{\mu}(0)\right| \chi\left(p_{i}\right)\right\rangle=\bar{u}\left(p_{f}\right) \Gamma_{\chi}^{\mu}(q) u\left(p_{i}\right),
$$

where $p_{i, f}$ and $q=p_{i}-p_{f}$ are four-momenta. For a neutral particle $\chi$ the vertex functions reads

$$
\Gamma_{\chi}^{\mu}(q)=i \sigma^{\mu \nu} q_{\nu}\left(\mu_{\chi}+i d_{\chi} \gamma^{5}\right)+\left(q^{2} \gamma^{\mu}-q^{\mu} q\right)\left(b_{\chi}-a_{\chi} \gamma^{5}\right) .
$$

Here we regard the various moments as being generated at an energy scale well above the energies involved in the stellar production; they are hence $q$-independent.

\section{STELLAR OBSERVABLES}

In this section we review the arguments on stellar energy loss. Active stars such as RG, HB, or the Sun are systems of negative heat capacity: if energy is lost, either through photon emission or through new, anomalous processes, the decrease of total energy causes the gravitational energy to become more negative. By virtue of the virial theorem, the average kinetic energy and thereby the photon temperature increases. The system heats up leading to a faster consumption of its nuclear fuel while the overall stellar structure remains largely unchanged. In contrast, dead stars such as white dwarfs or the proto-neutron star formed in core-collapse SN are supported by degeneracy pressure and stellar energy loss implies a cooling of the system.
Constraints are then derived based on an observationally inferred cooling curve.

\section{A. RG and HB stars}

In globular clusters (GCs), the population of stars on the red giant branch vs horizontal branch is directly related to the lifetime of stars in the respective phases. Their observationally inferred number ratio agrees with standard predictions to within $10 \%$. Anomalous energy losses shorten the helium-burning lifetime in HB stars, creating an imbalance in the number of $\mathrm{HB}$ vs RG stars. This constrains the luminosity in non-standard channels to be less than approximately $10 \%$ of the standard heliumburning luminosity of the HB core [3],

$$
\int_{\text {core }} d V \dot{Q}<10 \% \times L_{\mathrm{HB}} \quad(\mathrm{HB})
$$

Following [3], $L_{\mathrm{HB}}$ will be taken as $20 L_{\odot}$ for a $0.5 M_{\odot}$ core below. The values of the Solar mass and luminosity are $M_{\odot}=1.99 \times 10^{33} \mathrm{~g}$ and $L_{\odot}=3.83 \times 10^{33} \mathrm{erg} / \mathrm{s}$, respectively. The computation of the anomalous energy loss rate per unit volume and time, $\dot{Q}$, will be the subject of the next section.

A constraint for RG stars may be derived from an agreement between predicted and observationally inferred core masses prior to helium ignition. Energy loss delays the latter and the core mass keeps increasing as the hydrogen burning "ashes" fall onto the degenerate $\mathrm{He}$ core. Preventing an increase in core mass by no more than $5 \%$ yields the constraint [3],

$$
\dot{Q}<10 \mathrm{erg} / \mathrm{g} / \mathrm{s} \times \rho \quad(\mathrm{RG}) .
$$

Here, $\dot{Q}$ is to be evaluated at an average density of $\rho=$ $2 \times 10^{5} \mathrm{~g} / \mathrm{cm}^{3}$ and a temperature of $T=10^{8} \mathrm{~K} \simeq 8.6 \mathrm{keV}$, slightly higher than that of HB stars.

The criterion (8) on energy loss can be improved utilizing high precision photometric observations of GCs. For example, considering the brightness of the tip of the RG branch, [43] has provided a detailed error budget, and new limits on neutrino dipole moments from GC M5 were derived based on predictions of absolute brightness in the presence of anomalous energy loss that are obtained with dedicated stellar evolutionary codes. It was found, however, that previously derived limits based on (8) remain largely intact, as there appears to be a slight preference for anomalous energy loss channels [43]. In the following, for our purposes it will hence be entirely sufficient to employ the simple condition (8) to arrive at constraints on the EM form factors. 


\section{B. Sun}

Solar neutrino fluxes are a direct measure of the nuclear fusion rates inside the Sun. For example, not only the ${ }^{8} \mathrm{~B}$ neutrino flux is very well measured but also the sensitive dependence of the responsible reaction on the temperature provides an excellent handle for constraining anomalous energy losses. The ensuing constraint is then phrased in terms of the total Solar photon luminosity $[44,45]$, as

$$
\int_{\text {Sun }} d V \dot{Q}<10 \% \times L_{\odot} \quad(\text { Sun }) .
$$

It is important to note that (9) is basically insensitive to the long-standing "solar opacity problem": the measured ${ }^{8} \mathrm{~B}$ neutrino flux is situated in the overlap region of the nominal error ranges between the discrepant high- and lowmetallicity determinations of the Solar chemical composition [8]; see the respective Refs. [46,47]. Hence, (9) suffices as a criterion, awaiting further developments on Solar opacity determinations.

\section{Supernova}

New particles that are emitted from the proto-neutron star and that stream freely may quench the electroweak rates of neutrino emission during the cooling phase. The involved processes and their dynamics are highly complex. However, an approximate but very useful criterion to constrain additional energy loss is the condition that the total luminosity due to nonstandard processes should not exceed the neutrino luminosity at one second after core bounce [3],

$$
\int_{\text {core }} d V \dot{Q}<L_{\nu}=3 \times 10^{52} \mathrm{erg} / \mathrm{s} \quad(\mathrm{SN}) .
$$

The applicability of the bounds above are contingent on that the SN1987A was a neutrino-driven SN explosion ${ }^{1}$ and that the produced particles are able to escape the dense environment of the SN remnant, assumed to be a protoneutron star (PNS). Below, we will account for this socalled "trapping-limit" in the case of SN. For all other systems introduced above, trapping is either irrelevant or happens in a parameter region that is excluded otherwise.

\section{PRODUCTION CROSS SECTIONS AND ENERGY LOSS RATES}

In this section we first provide the general formula for $\chi \bar{\chi}$ pair production in the thermal bath, before breaking it down into the most relevant pieces that dominate the in-medium production cross sections and, thereby, the stellar cooling rates.

\footnotetext{
${ }^{1}$ For an alternative explosion mechanism where the SN1987A bounds would not apply, see $[48,49]$.
}

\section{A. Exact formula for $\chi \bar{\chi}$ pair production}

In thermal field theory, the production rate of a decoupled fermion per volume per time may be obtained from its relation to the imaginary part of its self-energy in medium [50] via

$$
\dot{N}_{\chi}=-\int \frac{d^{3} \vec{p}_{\chi}}{(2 \pi)^{3}} \frac{1}{\left(e^{E_{\chi} / T}+1\right)} \frac{\operatorname{Im} \Pi_{\chi}\left(E_{\chi}, \vec{p}_{\chi}\right)}{E_{\chi}},
$$

where $\operatorname{Im} \Pi_{\chi}\left(E_{\chi}, \vec{p}_{\chi}\right)=\bar{u}\left(p_{\chi}\right) \Sigma\left(E_{\chi}, \vec{p}_{\chi}\right) u\left(p_{\chi}\right)$ is the discontinuity of the thermal self-energy of $\chi, \Sigma\left(E_{\chi}, \vec{p}_{\chi}\right)$; $u\left(p_{\chi}\right)$ and $\bar{u}\left(p_{\chi}\right)$ are free particle spinors with fourmomentum $p_{\chi}=\left(E_{\chi}, \vec{p}_{\chi}\right)$. To lowest order in the dark coupling, $\Sigma\left(E_{\chi}, \vec{p}_{\chi}\right)$ is found from the one-loop diagram with a dressed photon propagator attached to the $\chi$ fermion line. A general exposition on calculating discontinuities in the thermal plasma is found in [50,51].

Below, in Eq. (13), we are using a different formulation, and the equivalence may be appreciated in the following way: when cutting the self-energy diagram for $\chi$, the optical theorem implies that the production rate may also be obtained by computing all graphs where a photon $\gamma^{*}$ of four-momentum $k=p_{\chi}+p_{\bar{\chi}}$ emerges from a SM current and is being dotted into the dark current of the $\chi \bar{\chi}$ pair. The SM-process that leads to the creation of $\gamma^{*}$ is in turn related to the imaginary part of the photon self-energy in the medium, $\operatorname{Im} \Pi_{\mu \nu}$, where

$$
\Pi^{\mu \rho}=\left(\epsilon_{\mathrm{T}, 1}^{\mu} \epsilon_{\mathrm{T}, 1}^{\rho}+\epsilon_{\mathrm{T}, 2}^{\mu} \epsilon_{\mathrm{T}, 2}^{\rho}\right) \Pi_{\mathrm{T}}+\epsilon_{\mathrm{L}}^{\mu} \epsilon_{\mathrm{L}}^{\rho} \Pi_{\mathrm{L}} .
$$

Here $\epsilon_{\mathrm{T}, \mathrm{L}}$ are the transverse and longitudinal photon polarization vectors and $\Pi_{\mathrm{L}, \mathrm{T}}(k)$ is thermal photon selfenergy for the respective polarization; explicit expressions are given in Appendix A. Identifying the leading contributions to $\operatorname{Im} \Pi_{\mathrm{L}, \mathrm{T}}(k)$ in various mediums then allows us to account for the dominant $\chi$ pair production channels.

The exact differential production rate per volume of $\chi \bar{\chi}$ pairs via a photon of 4-momentum $k=(\omega, \vec{k})$ emerging from any SM process to lowest order in the dark current can be obtained by borrowing the results from dilepton production in hot matter; see e.g., [52,53]. Adopted to our purposes (see Appendix B) it reads

$$
\begin{aligned}
\frac{d \dot{N}_{\chi}}{d s_{\chi \bar{\chi}}}= & -\sum_{i=\mathrm{T}, \mathrm{L}} g_{i} \int \frac{d^{3} \vec{k}}{(2 \pi)^{3}} \frac{1}{\left(e^{\omega / T}-1\right)} \frac{\operatorname{Im}_{i}(\omega, \vec{k})}{\omega} \\
& \times \frac{f\left(s_{\chi \bar{\chi}}\right)}{16 \pi^{2}\left|s_{\chi \bar{\chi}}-\Pi_{i}\right|^{2}} \sqrt{1-\frac{4 m_{\chi}^{2}}{s_{\chi \bar{\chi}}}},
\end{aligned}
$$

where $s_{\chi \bar{\chi}}=k^{2}$ is the invariant mass of the $\chi$-pair and the internal degrees of freedom (d.o.f.) of two polarization modes are $g_{\mathrm{T}}=2, g_{\mathrm{L}}=1$. The differences in the various interaction possibilities are entirely captured in a factor that 


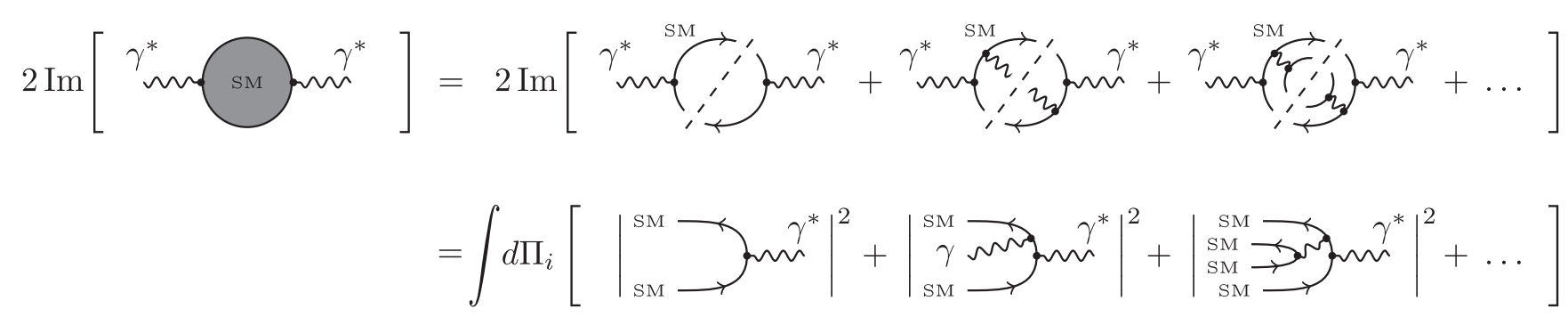

FIG. 2. Optical theorem relating the imaginary part of the photon self energy to the sum of all SM processes that create an off shell photon $\gamma^{*}$. The first equality shows the leading individual contributions to the self-energy. When the latter loop-diagrams are cut, they correspond to the scattering processes shown in the second line, where $d \Pi_{i}$ symbolizes the phase space integral of all external particles, except $\gamma^{*}$. When the scattering diagrams are deformed in a way such that two SM particles are in the initial state, the processes correspond to annihilation, Compton scattering and bremsstrahlung (from left to right). Any diagrams with $\chi$ particles involved yield contributions to the production rate (13) that are of higher order in the dark coupling.

will repeatedly appear and that was obtained in our preceding work [30],

$$
\begin{aligned}
& \mathrm{MDM}: f\left(s_{\chi \bar{\chi}}\right)=\frac{2}{3} \mu_{\chi}^{2} s_{\chi \bar{\chi}}^{2}\left(1+\frac{8 m_{\chi}^{2}}{s_{\chi \bar{\chi}}}\right), \\
& \mathrm{EDM}: f\left(s_{\chi \bar{\chi}}\right)=\frac{2}{3} d_{\chi}^{2} s_{\chi \bar{\chi}}^{2}\left(1-\frac{4 m_{\chi}^{2}}{s_{\chi \bar{\chi}}}\right), \\
& \mathrm{AM}: f\left(s_{\chi \bar{\chi}}\right)=\frac{4}{3} a_{\chi}^{2} s_{\chi \bar{\chi}}^{3}\left(1-\frac{4 m_{\chi}^{2}}{s_{\chi \bar{\chi}}}\right), \\
& \mathrm{CR}: f\left(s_{\chi \bar{\chi}}\right)=\frac{4}{3} b_{\chi}^{2} s_{\chi \bar{\chi}}^{3}\left(1+\frac{2 m_{\chi}^{2}}{s_{\chi \bar{\chi}}}\right) .
\end{aligned}
$$

Equation (13) is the general expression of the weakly coupled $\chi$ pair-production rate from the thermal medium; details are found in Appendix B.

The contribution to $\chi \bar{\chi}$ production to leading order in $\alpha$ is given by the pole in (13), i.e., for $s_{\chi \bar{x}}=\operatorname{Re} \Pi_{\mathrm{L}, \mathrm{T}}$. When this condition is met, (13) reduces to the decay rate of thermal photons $\gamma_{\mathrm{L}, \mathrm{T}} \rightarrow \chi \bar{\chi}$. Hence, resonant $\chi \bar{\chi}$ production is fully accounted for by $\gamma_{\mathrm{L}, \mathrm{T}}$ decay. The decay itself becomes possible by virtue of the in-medium (squared) mass of $\gamma_{\mathrm{L}, \mathrm{T}}$ : it is given by $\operatorname{Re} \Pi_{\mathrm{L}, \mathrm{T}}\left(\omega_{\mathrm{L}, \mathrm{T}}, \vec{k}\right)$, where $\omega_{\mathrm{L}, \mathrm{T}}$ denotes the solution of $\omega(|\vec{k}|)$ of the corresponding longitudinal and transverse dispersion relations $\omega^{2}-|\vec{k}|^{2}-\operatorname{Re} \Pi_{\mathrm{L}, \mathrm{T}}(\omega, \vec{k})=0$. Plasmon decay is discussed in the following subsection, and explicitly calculated in (B7)-(B9) in Appendix B. The expressions for $\operatorname{Re} \Pi_{\mathrm{L}, \mathrm{T}}$ and finite-temperature dispersion relations are found in (A5)-(A6).

Production off-the-pole to Eq. (13) can be elucidated by studying the contributions to Im $\Pi$ using the optical theorem, illustrated in Fig. 2. The left-hand side shows the fully dressed vacuum polarization of an off shell photon $\gamma^{*}$, found by considering loop-diagrams of increasing order in $\alpha$ illustrated in the first equality. When those loop diagrams are cut, their imaginary parts are given by the tree-level production processes for $\gamma^{*}$ shown in the last equality. The leading $\alpha$ contribution to $\mathrm{Im} \Pi$ is then given by the electron one-loop diagram. Although it is well known that on shell plasmon decay $\gamma_{\mathrm{L}, \mathrm{T}} \rightarrow e^{+} e^{-}$remains forbidden at finite temperature [54], an electron loop still contributes to $\operatorname{Im} \Pi$ in the off shell case. The associated process is then $e^{+} e^{-}$annihilation to $\chi \bar{\chi}$, i.e., process $(2 \mathrm{~b})$.

The second and third diagrams in the last line of Fig. 2 are related to $\chi \bar{\chi}$ production in Compton scattering and bremsstrahlung. Here, it is important to note that $s_{\chi \bar{x}}=$ $\mathrm{Re} \Pi_{\mathrm{L}, \mathrm{T}}$ can also be met in the photon propagator that produces the $\chi$-pair with invariant squared mass $s_{\chi \bar{x}}$. However, including such resonances would amount to double-counting. As we have seen above, the pole contributions are already captured by plasmon decay. ${ }^{2}$ In our calculations, we explicitly avoid this situation by setting $\Pi_{\mathrm{L}, \mathrm{T}} \rightarrow 0$ in the propagator if the resonance is kinematically allowed for the photon that directly couples to the dark current. We have numerically verified that our results remain otherwise unaffected by neglecting the thermal shift in the photon propagator.

Finally, there is also a potential double counting between Compton scattering and bremsstrahlung processes, which happens when in the bremsstrahlung process the photon exchanged between two initial particles carries 4-momentum $q$ [see Fig. 1(d)] that satisfies the dispersion relation $q^{2}-\operatorname{Re}_{L}\left(q^{0}, \vec{q}\right)=0$, leading to the exchange of an on shell longitudinal plasmon. The process then becomes equivalent to Compton scattering $e / N+\gamma_{\mathrm{L}} \rightarrow e / N+\chi+\bar{\chi}$. This has been reported for axion production processes, where the contribution of latter is mostly covered by that of bremsstrahlung [57]. To avoid such double-counting, we take the static approximation $\left(q^{0}=0\right)$ for the thermal mass

\footnotetext{
${ }^{2} \mathrm{~A}$ heuristic argument on such double counting was also given in the context of neutrino pair emission in Sec. 2.5 of [55]. It furthermore appears to us, that double counting may have occurred in [56] where a potentially resonant bremsstrahlung process was added to the plasmon decay contribution.
} 
of the photon exchanged in bremsstrahlung processes, which is a valid limit as the nucleon mass is large. As $q^{2}<0$ and $\Pi_{L}\left(q^{0}=0, \vec{q}\right)$ is always positive, the exchanged photon can not become on shell in bremsstrahlung processes, thus double counting is avoided (see Sec. IV D for more details).

\section{B. $\gamma_{\mathrm{T}, \mathrm{L}}$ decay}

The on shell process of photon decay to $\chi \bar{\chi}$ [Fig. 1(a)] becomes possible in the medium and has an important analogy in the literature, the plasmon decay to neutrinos. Since the dispersion relation for transverse and longitudinal thermal photons are distinct, it is again helpful to separate the two polarizations in the calculation. Explicitly, we obtain for the decay rate per d.o.f.,

$$
\Gamma_{\mathrm{T}, \mathrm{L}}=\frac{1}{16 \pi} Z_{\mathrm{T}, \mathrm{L}} \sqrt{1-\frac{4 m_{\chi}^{2}}{\omega_{\mathrm{T}, \mathrm{L}}^{2}-|\vec{k}|^{2}}} \frac{f\left(\omega_{\mathrm{T}, \mathrm{L}}^{2}-|\vec{k}|^{2}\right)}{\omega_{\mathrm{T}, \mathrm{L}}},
$$

where $\omega_{\mathrm{T}, \mathrm{L}}=\omega_{\mathrm{T}, \mathrm{L}}(|\vec{k}|)$ for each polarization mode, as defined above. Details on the definition of the wave function renormalization factors $Z_{\mathrm{T}, \mathrm{L}}$ and the calculation are again given in Appendix B. In the limit of $m_{\chi} \rightarrow 0$, the decay widths for MDM agree with the well-known formulas for plasmon decay to a neutrino pair [3].

For the plasmon decay processes, the energy loss rate can be expressed as [3]

$$
\begin{aligned}
& \dot{Q}_{\text {decay, } \mathrm{T}}=\frac{2}{2 \pi^{2}} \int_{0}^{\infty} d|\vec{k}| \frac{|\vec{k}|^{2} \Gamma_{\mathrm{T}} \omega_{\mathrm{T}}}{e^{\omega_{\mathrm{T}} / T}-1} \Theta\left(\omega_{\mathrm{T}}^{2}-|\vec{k}|^{2}-4 m_{\chi}^{2}\right), \\
& \dot{Q}_{\text {decay }, \mathrm{L}}=\frac{1}{2 \pi^{2}} \int_{0}^{k_{\max }} d|\vec{k}| \frac{|\vec{k}|^{2} \Gamma_{\mathrm{L}} \omega_{\mathrm{L}}}{e^{\omega_{\mathrm{L}} / T}-1} \Theta\left(\omega_{\mathrm{L}}^{2}-|\vec{k}|^{2}-4 m_{\chi}^{2}\right) .
\end{aligned}
$$

The expression for $k_{\max }$ is given in Eq. (A7). For a nonrelativistic medium (HB, RG, Sun), the dispersion relation crosses the light cone at $|\vec{k}|=k_{\max }$, signaling the damping of longitudinal modes (i.e., Landau damping); for a relativistic plasma (SN) $k_{\max } \rightarrow \infty$. The relative factor of 2 between the expressions reflects the counting of polarization d.o.f. Finally, the last factor is a kinematic restriction on the phase space, $\omega_{T, L}^{2}-|\vec{k}|^{2} \geq 4 m_{\chi}^{2}$. For transverse mode thermal photons, the integral becomes bounded from below since $\omega_{T}^{2}-|\vec{k}|^{2}$ increases as $|\vec{k}|$ increases according to the dispersion relation. For the longitudinal case, the integral is additionally bounded from above since the trend in $\omega_{\mathrm{L}}^{2}-|\vec{k}|^{2}$ with respect to $|\vec{k}|$ is reversed.

\section{C. $e^{+} e^{-}$annihilation}

The degenerate plasma of the PNS core with temperature $T \gg m_{e}$ contains a population of $e^{+}$, allowing for dark state pair-production through $e^{+} e^{-}$annihilation [Fig. 1(b)]. The calculation for the pair production cross section is detailed in Appendix B.

In terms of the invariant $s=\left(p_{1}+p_{2}\right)^{2}$ and the sum/ difference of incoming $e^{\mp}$ energies $E_{1,2}$ in the frame of the thermal bath, i.e., $E_{ \pm} \equiv E_{1} \pm E_{2}$, the corresponding cross section mediated by the transverse polarization part of the propagator reads

$$
\sigma_{\mathrm{T}}=\frac{\alpha\left[s E_{-}^{2}+\left(4 m_{e}^{2}+s\right)\left(E_{+}^{2}-s\right)\right]}{8 \sqrt{s\left(s-4 m_{e}^{2}\right)}\left(E_{+}^{2}-s\right)\left(s-\Pi_{T}\right)^{2}} \sqrt{1-\frac{4 m_{\chi}^{2}}{s}} f(s) .
$$

For the longitudinal part we obtain

$$
\sigma_{\mathrm{L}}=\frac{\alpha\left[s\left(E_{+}^{2}-E_{-}^{2}-s\right)\right]}{8 \sqrt{s\left(s-4 m_{e}^{2}\right)}\left(E_{+}^{2}-s\right)\left(s-\Pi_{L}\right)^{2}} \sqrt{1-\frac{4 m_{\chi}^{2}}{s}} f(s) .
$$

Note that there is no interference term between the two. Furthermore, the sum of both cross sections, $\sigma_{\mathrm{T}}+\sigma_{\mathrm{L}}$, becomes Lorentz invariant in the limit of $\Pi_{T, \mathrm{~L}} \rightarrow 0{ }^{3}$

Before using (17) and (18) in the calculation of the energy loss rate, a comment on the analytic structure is in order. Although it appears that the process may be significantly enhanced when $s=\operatorname{Re} \Pi_{\mathrm{T}, \mathrm{L}}$, this condition is never met: for the same reason that the decay of thermal photons into an electron-positron pair $\left(\gamma_{\mathrm{T}, \mathrm{L}} \rightarrow e^{+} e^{-}\right)$is forbidden [54], the finite-temperature corrections to $m_{e}$ prevent the process (2) from going on shell. It is for this reason that we have explicitly evaluated the thermal electron mass for the employed radial profile of the PNS; see Appendix A. In other words, we use a thermal electron mass in $\mathrm{SN}$, and use the zero-temperature electron mass in $\mathrm{HB}, \mathrm{RG}$ and Sun, where $e^{+} e^{-}$annihilation is of little relevance. The values of chemical potential $\mu_{e}$ are selfconsistently adjusted to match the numerical PNS profiles from the literature (see below).

The energy loss rate of $e^{+} e^{-}$annihilation is found by weighing the emission process by the total radiated final state energy $E_{3}+E_{4}=E_{1}+E_{2}$ and by the probability of finding the initial states with the respective energies $E_{1}$ and $E_{2}$,

$$
\begin{aligned}
\dot{Q}_{\mathrm{ann}}= & \int d \Pi_{i=1,2,3,4}(2 \pi)^{4} \delta^{4}\left(p_{1}+p_{2}-p_{3}-p_{4}\right) \\
& \times g_{e^{-}} g_{e^{+}} f_{e^{-}} f_{e^{+}} \frac{1}{4} \sum_{\text {spins }}\left|\mathcal{M}_{\mathrm{ann}}\right|^{2}\left(E_{1}+E_{2}\right) .
\end{aligned}
$$

\footnotetext{
${ }^{3}$ We use a definition of the cross section for which the Møller velocity instead of the relative velocity $\left|\vec{v}_{1}-\vec{v}_{2}\right|$ appears. At zero temperature, this makes the cross section a Lorentz invariant quantity; see the discussion in [58].
} 
Here, $f_{e^{ \pm}}$are the phase-space distributions of $e^{ \pm}$, with internal d.o.f. $g_{e^{ \pm}}=2$, and $\left|\mathcal{M}_{\text {ann }}\right|^{2}$ is the squared matrix element for $e^{+} e^{-}$annihilation into the dark state pair. In (19) a Pauli-blocking factor induced by $\chi$ and $\bar{\chi}$ is neglected; we have verified that this does not affect the derived constraints. Finally, $d \Pi_{i}=\prod_{i} d^{3} \vec{p}_{i}(2 \pi)^{-3}\left(2 E_{i}\right)^{-1}$ is the Lorentz invariant phase space element.

The energy loss rate can be written in terms of the cross sections $\sigma_{\mathrm{T}, \mathrm{L}}$. Borrowing from the discussion on phase space in [59], we find explicitly

$$
\begin{aligned}
\dot{Q}_{\mathrm{ann}}= & \int_{4 m_{\mathrm{th}}^{2}}^{\infty} d s \int_{\sqrt{s}}^{\infty} d E_{+} \int_{-\sqrt{\left(1-4 m_{e}^{2} / s\right)\left(E_{+}^{2}-s\right)}}^{\sqrt{\left(1-4 m_{e}^{2} / s\right)\left(E_{+}^{2}-s\right)}} d E_{-} \\
& \times \frac{1}{64 \pi^{4}} g_{e^{-}} g_{e^{+}} f_{e^{-}} f_{e^{+}} E_{+} \sqrt{s\left(s-4 m_{e}^{2}\right)} \sigma_{\mathrm{T}, \mathrm{L}} .
\end{aligned}
$$

The distribution functions $f_{e^{-}}$and $f_{e^{+}}$read

$$
f_{e^{\mp}}=\frac{1}{e^{\left(E_{+} \pm E_{-} \mp 2 \mu_{e}\right) / 2 T}+1} .
$$

Here, $\mu_{e}$ is the chemical potential of electrons and $T$ is the temperature. The threshold mass $m_{\text {th }}$ is equal to $\max \left\{m_{e}, m_{\chi}\right\}$.

\section{Compton scattering}

For $2 \rightarrow 3$ Compton scattering $\left(e^{-} / N+\gamma_{\mathrm{T}, \mathrm{L}} \rightarrow e^{-} /\right.$ $N+\chi+\bar{\chi}$ ) with an initial $\gamma_{\mathrm{T}, \mathrm{L}}$ [Fig. 1(c)], we calculate the differential cross section via

$$
\frac{d \sigma_{2 \rightarrow 3}}{d s_{\chi \bar{\chi}}}=\sigma_{2 \rightarrow 2}\left(s_{\chi \bar{\chi}}\right) \frac{f\left(s_{\chi \bar{\chi}}\right)}{16 \pi^{2} s_{\chi \bar{\chi}}^{2}} \sqrt{1-\frac{4 m_{\chi}^{2}}{s_{\chi \bar{\chi}}}} .
$$

Here, $\sigma_{2 \rightarrow 2}\left(s_{\chi \bar{x}}\right)$ is the cross section of the two-body Compton scattering with the final-state photon having a mass $\sqrt{s_{\chi \bar{x}}}$. We are only required to consider the process on electrons, $e^{-}+\gamma_{\mathrm{T}, \mathrm{L}} \rightarrow e^{-}+\chi+\bar{\chi}$, as Compton scattering on protons is strongly suppressed. Following the treatment in [55] and our discussion above, we neglect the thermal mass of the final state photon in $\sigma_{2 \rightarrow 2}$ to avoid any potential double counting with $\gamma_{T, L}$ decay. For the initial state photon in the integration of energy loss rate, Eq. (23) below, the thermal mass is properly taken in account through the dispersion relation (A6).

Furthermore, note that there is no double counting between the Compton process and bremsstrahlung either in our treatment. A double counting would appear if the $t$-channel photon exchange in bremsstrahlung, with 4-momentum $q$ [see Fig. 1(d)], goes on resonance. This is in principle possible for the longitudinal mode, since $\Pi_{L}$ in the propagator could become negative once the dispersion relation of $\gamma_{\mathrm{L}}$ crosses the light cone. Nevertheless, in the electron bremsstrahlung process discussed below-most relevant for RG, HB and the Sun - the proton recoil and hence the energy exchange are extremely small. Therefore, the propagator can be taken in the static limit (energy exchange $q^{0} \rightarrow 0$ ). This limit amounts to Debye screening, characterized by $\Pi_{\mathrm{L}}\left(q^{0} \rightarrow 0,|\vec{q}|\right)$. Since the screening scale is always positive, a resonance is never met. Therefore, we include the contribution from $e^{-}+\gamma_{\mathrm{L}} \rightarrow e^{-}+\chi+\bar{\chi}$ to capture the $t$-channel resonance contribution of electron bremsstrahlung, although it is less important than plasmon decay.

The energy loss rate from Compton scattering is calculated in a similar way as (19), but here $E_{\text {loss }}$ is given by the energy carried by the virtual photon in the medium frame,

$$
\begin{aligned}
\dot{Q}_{\text {Compton }}= & \int d \Pi_{i=1,2} 4 E_{1} E_{2} \sigma_{2 \rightarrow 3}^{\mathrm{T}, \mathrm{L}} v_{M} g_{e^{-}} g_{\mathrm{T}, \mathrm{L}} f_{1} f_{2} \\
& \times\left(1-f_{3}\right) E_{\mathrm{loss}},
\end{aligned}
$$

where $f_{1,2,3}$ are the distribution functions of the incoming electron, $\gamma_{\mathrm{T}, \mathrm{L}}$ and outgoing electron, respectively, with $g_{e^{-}}=g_{\mathrm{T}}=2$ and $g_{\mathrm{L}}=1$ the internal d.o.f. for the incoming electron and $\gamma_{\mathrm{T}, \mathrm{L}}$. Pauli blocking is accounted for by including the factor $\left(1-f_{3}\right)$. The energy loss $E_{\text {loss }}=E_{\bar{\chi}}+E_{\chi}$ can be expressed in terms of variables defined in the medium frame. Moreover, for RG, HB and the Sun, the relativistic corrections induced by transforming from the center-of-mass $(\mathrm{CM})$ frame to the medium frame are very small and are neglected for simplicity.

\section{E. $e^{-} N$ bremsstrahlung}

In this subsection we consider dark state pair production from bremsstrahlung of electrons on protons or other nuclei [Fig. 1(d)]. Similar to the Compton scattering above, we also relate the $2 \rightarrow 4$ cross section to a $2 \rightarrow 3$ process of $e N \rightarrow e N+\gamma_{T, L}^{*}$ in which the emitted photon, $\gamma_{T, L}^{*}$, has an invariant mass $\sqrt{s_{\chi \bar{x}}}$,

$$
\frac{d \sigma_{2 \rightarrow 4}}{d s_{\chi \bar{\chi}}}=\sigma_{2 \rightarrow 3}\left(s_{\chi \bar{\chi}}\right) \frac{f\left(s_{\chi \bar{\chi}}\right)}{16 \pi^{2} s_{\chi \bar{\chi}}^{2}} \sqrt{1-\frac{4 m_{\chi}^{2}}{s_{\chi \bar{\chi}}}} .
$$

In the following we shall only consider photon-emission from electrons, as the emission from the nuclear leg is suppressed by a factor of $\left(Z m_{e} / m_{N}\right)^{2} \ll 1$ where $Z$ and $m_{N}$ are the charge and mass of the nucleon/nucleus. Furthermore, ordinary electron-electron bremsstrahlung is a quadrupole emission process and correspondingly smaller in practice. We therefore also neglect such production channel.

The $e N$ process is sensitive to the details of in-medium corrections. To this end, recall that the $t$-channel photon exchange in Fig. 1(d) has a well-known Coulomb divergence in the limit of vanishing momentum-transfer. This issue is mitigated by two factors: first, the divergence is not met kinematically as long as $m_{\chi} \neq 0$ since a minimum momentum transfer is necessary to create the final state pair. Second, the medium itself regulates the process 
through the Debye screening of bare charges characterized by a momentum scale $k_{D}$. The latter appears as the static limit of $\Pi_{\mathrm{L}}\left(q^{0} \rightarrow 0, \vec{q}\right)$ and for a classical plasma reads

$$
k_{D}^{2}=\frac{4 \pi \alpha n_{e}}{T}+\text { ion-contributions. }
$$

For the numerical results, we have calculated $\sigma_{2 \rightarrow 3}$ in (24) using the propagator (A4), neglecting, for simplicity, ion contributions. We separate the squared amplitude into transverse and longitudinal parts and include the static limits of $\Pi_{T, L}$ in the respective propagators. For the longitudinal part, the zero-temperature propagator $q^{-2}$ is replaced by $\left(q^{2}-k_{D}^{2}\right)^{-1}$. In contrast, there is no magnetic screening in the static limit $\left[\Pi_{\mathrm{T}}\left(q^{0} \rightarrow 0,|\vec{q}|\right)=0\right]$; hence there is no thermal screening for the propagator of the transverse mode. We find that in the nonrelativistic limit the contribution of the longitudinal mode dominates.

To avoid any double counting between this process and the $\gamma_{\mathrm{T}, \mathrm{L}}$ decay, we need to subtract the contribution when the virtual photon that directly couples to $\chi$ goes on shell. As stated above, this is achieved by setting $\Pi_{T, L}$ in the corresponding propagator to zero. Since this should overestimate the production rate at $s_{\chi \bar{x}} \leq \Pi_{\mathrm{T}, \mathrm{L}}$, we have also tested an opposite option of choosing $\Pi_{\mathrm{T}, \mathrm{L}} \rightarrow$ $-\Pi_{\mathrm{T}, \mathrm{L}}\left(E_{\chi}+E_{\bar{\chi}}\right)$ to avoid the singularity, which underestimates the production rate. We find that both prescriptions lead to same results at the percent level, which justifies our simplification of taking $\Pi_{\mathrm{T}, \mathrm{L}} \equiv 0$ for the photon that directly couples to $\chi$.

For dark state pair production in $e^{-}$bremsstrahlung on protons and nuclei, the energy loss rate is expressed as

$\dot{Q}_{\text {brem }}=\int d \Pi_{i=1,2} 4 E_{1} E_{2} \sigma_{2 \rightarrow 4} v_{M} g_{1} g_{2} f_{1} f_{2}\left(1-f_{3}\right) E_{\text {loss }}$,

where $f_{1,2,3}$ are the distribution functions of the incoming electron, proton/nucleus and outgoing electron, with $g_{1,2}$ the internal d.o.f. for the incoming particles. We have neglected the Pauli blocking factor for final-state protons/ nuclei as it plays little role. The Møller velocity $v_{M}=$ $F /\left(E_{1} E_{2}\right)$ is given in terms of the flux factor $F$ found in (B19). The energy carried-away by the dark states is $E_{\text {loss }}=E_{\bar{\chi}}+E_{\chi}$ and its expression in the medium frame is introduced in Appendix B.

Making the approximation that protons and other nuclei are at rest, their phase-space integral gives $\int d \Pi_{2} f_{2}=$ $n_{N} /\left(2 m_{N} g_{2}\right)$, where $n_{N}$ is the number density of the protons/nuclei. Hence we arrive at

$\dot{Q}_{\text {brem }}=\int_{m_{e}+2 m_{\chi}}^{\infty} d E_{1} \frac{2 n_{N} E_{1} E_{2} v_{M}}{(2 \pi)^{2} m_{N}}\left|\vec{p}_{1}\right| g_{1} f_{1} \sigma_{2 \rightarrow 4}\left(1-f_{3}\right) E_{\mathrm{loss}}$, with $\left|\vec{p}_{1}\right|=\sqrt{E_{1}^{2}-m_{e}^{2}}$ and where $\sigma_{2 \rightarrow 4}$ is obtained from integrating (24) over appropriate boundaries (see Appendix B 5). Generically, bremsstrahlung is less effective when pair annihilation or plasmon decay are open as production channels, but it can be dominant at low temperatures where the latter processes are kinematically suppressed.

Before ending this subsection, it is worth commenting on the so-called soft photon approximation, which states that in the limit that the emitted photon energy is small compared to the available kinetic energy (i.e., $\omega \ll E_{\text {kin }}$ ), the process of $e N \rightarrow e N+\gamma_{T, L}^{*}$ factorizes into a product of elastic scattering times a factor describing the additional emission of $\gamma_{T, L}^{*}$. While this approximation works well for the emission of a massless photon, it breaks down if the off shell photon's effective mass is large, $\sqrt{s_{\chi \bar{x}}} \sim E_{\mathrm{kin}}$. Overall, we find that the soft photon approximation describes the $2 \rightarrow 4$ process well for small $m_{\chi}$ in the nonrelativistic limit. However, for $2 m_{\chi} \sim E_{\text {kin }}$ or for relativistic initial states the approximation fails, and it is ultimately related to the UVsensitivity of the cross section (see Appendix C for details). Even though calculations simplify considerably in the soft photon limit, it cannot be applied for the whole $m_{\chi}$-range in electron bremsstrahlung, and we therefore calculate $\sigma_{2 \rightarrow 4}$ exactly, relegating details of the calculation to Appendix B. However, we will use the soft photon approximation in its region of validity to estimate the energy loss from nucleon bremsstrahlung in the next subsection.

\section{F. Nucleon bremsstrahlung}

Proton and nuclear bremsstrahlungs are strongly suppressed in low-temperature environments due to the negligible thermal velocities of the initial states. However, in the interior of a PNS, the typical nucleon velocity is $v \lesssim 1 / 3$ and $N N$-bremsstrahlung contributes to the total energy loss.

The photon that pair-creates the dark states is emitted from the proton-leg in proton-proton $(p p)$ and neutronproton $(n p)$ scattering. Radiating off the neutron-leg through the neutron magnetic dipole moment is suppressed. In addition, since $p p$-scattering is associated with quadrupole radiation, it is suppressed with respect $n p$ scattering by a factor $v^{2}$ [60]. Therefore, we only consider $n p$-scattering in the following. The interaction of protons and neutrons is mostly mediated by pions whose mass is of the order of the average momentum transfer in elastic collisions in PNS, allowing for a separation of the phase space into an elastic and an emission piece (see Appendix $\mathrm{C}$ for details). The energy loss rate in the nonrelativistic, nondegenerate limit ${ }^{4}$ is then given by [60]

\footnotetext{
${ }^{4}$ The corrections to Eq. (28) due to matter degeneracy in PNS are estimated in Ref. [60] to be small $(\approx 30 \%)$ compared to the corrections neglected in the soft-photon approximation, which are up to a factor of 3 as mentioned in Appendix C.
} 


$$
\begin{aligned}
\dot{Q}_{n p}= & \frac{n_{n} n_{p}}{\sqrt{\pi}\left(m_{n} T\right)^{3 / 2}} \int_{2 m_{\chi}}^{\infty} d E_{\mathrm{kin}} E_{\mathrm{kin}}^{2} e^{-\frac{E_{\mathrm{kin}}}{T}} \int_{4 m_{\bar{\chi}}^{2}}^{E_{\mathrm{kin}}^{2}} d s_{\chi \bar{\chi}} \\
& \times \sqrt{1-\frac{4 m_{\chi}^{2}}{s_{\chi \bar{\chi}}}} \frac{f\left(s_{\chi \bar{\chi}}\right)}{16 \pi^{2} s_{\chi \bar{\chi}}^{2}} \sigma_{n p}^{T}\left(E_{\mathrm{kin}}\right) \mathcal{I}_{\omega}\left(s_{\chi \bar{\chi}}\right),
\end{aligned}
$$

where $n_{n}$ and $n_{p}$ are the neutron and proton number densities, $m_{n}$ is the average nucleon mass and $E_{\text {kin }}$ the available kinetic center of mass energy. For the elastic $n p$ scattering transport cross section $\sigma_{n p}^{T}$ we use the numerical data from Fig. 3 in Ref. [60] for $E_{\text {kin }} \gtrsim 1 \mathrm{MeV}$ and Fig. 2 in Ref. [61] for smaller energies; the emission piece $\mathcal{I}_{\omega}$ is given by Eq. (C8).

The energy loss rates from nucleon bremsstrahlung are comparable with the rates from plasmon decay and are depicted for $m_{\chi}=1 \mathrm{MeV}$ as a function of the PNS radius in Fig. 4.

\section{CONSTRAINTS ON THE EFFECTIVE COUPLING}

After calculating the energy loss rates induced by those relevant processes in each stellar environment (e.g., see Figs. 3-4), we apply the luminosity criteria introduced in Sec. III to obtain the upper bounds on the EM form-factors of light dark states.

\section{A. Limits from RG, HB, and the Sun}

In this subsection, we derive the constraints coming from HB and RG stars utilizing the above calculated anomalous

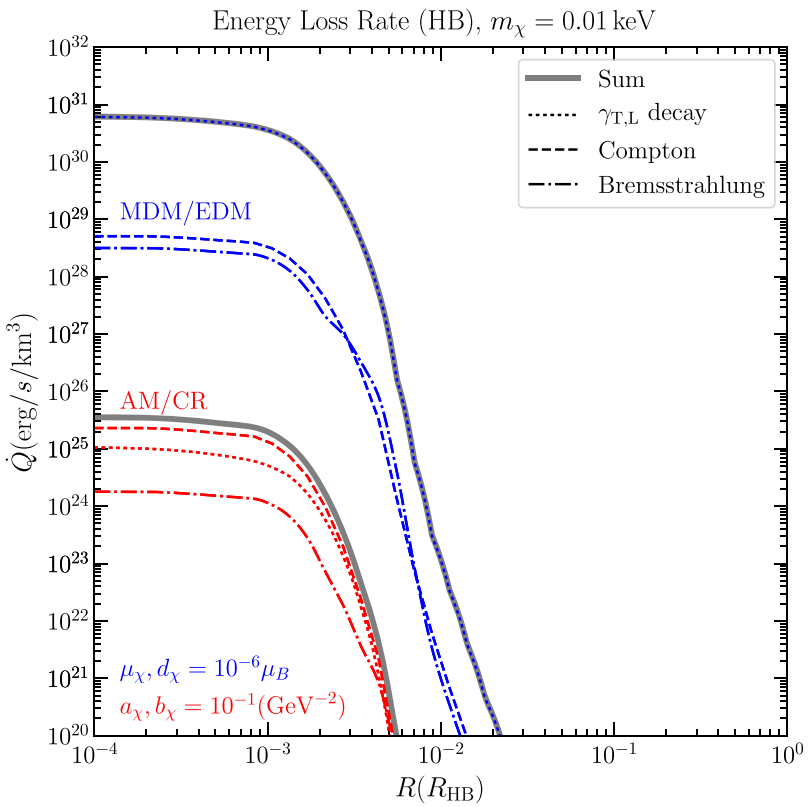

energy loss rates. For $\mathrm{HB}$, we consider a representative star of $0.8 M_{\odot}$ and utilize the stellar profiles for density, temperature and chemical partition between hydrogen and helium from [3,62], reproduced in Fig. 7 in Appendix A. The luminosity of its helium-burning core is $L_{\mathrm{HB}}=20 L_{\odot}$ to which (7) is then applied. For RG we use the prescription detailed below (8): a $0.5 M_{\odot}$ helium core with a constant density of $\rho=2 \times 10^{5} \mathrm{~g} / \mathrm{cm}^{3}$ and a temperature $T=10^{8} \mathrm{~K}$.

For the Sun, we use the standard Solar model BP05(OP) [63] to calculate the total power radiated into $\chi \bar{\chi}$ which in turn is constrained from (9). For bremsstrahlung we take the contribution of electron scattering on $\mathrm{H},{ }^{4} \mathrm{He}$ and other less abundant nuclei $\left({ }^{3} \mathrm{He}, \mathrm{C}, \mathrm{N}\right.$, and $\left.\mathrm{O}\right)$. For simplicity, we assume all targets are in a fully ionized state. We find numerically that the contribution from the second class of elements contribute $10 \%$ of the total energy loss rate from bremsstrahlung, as the coherent enhancement from atomic charge number $Z$ somewhat compensates for their scarcity in number.

The energy loss rates as a function of fractional stellar radius for $\mathrm{HB}$ (Sun) for all operators considered in (5) and (6) are shown in the left (right) panel of Fig. 3 for $m_{\chi}=10 \mathrm{eV}$ and $\mu_{\chi}\left(\right.$ or $\left.d_{\chi}\right)=10^{-6} \mu_{B}$ and $a_{\chi}\left(\right.$ or $\left.b_{\chi}\right)=0.1 \mathrm{GeV}^{-2}$. MDM and EDM as well as AM and CR lines essentially yield identical results. This is owed to the fact that production proceeds in the kinematically unsuppressed region $T \gg m_{\chi}$ for which the energy loss rates match; the $\gamma^{5}$ factor discriminating the interactions of same mass-dimension only plays a role when $\chi$ particles become nonrelativistic, hence

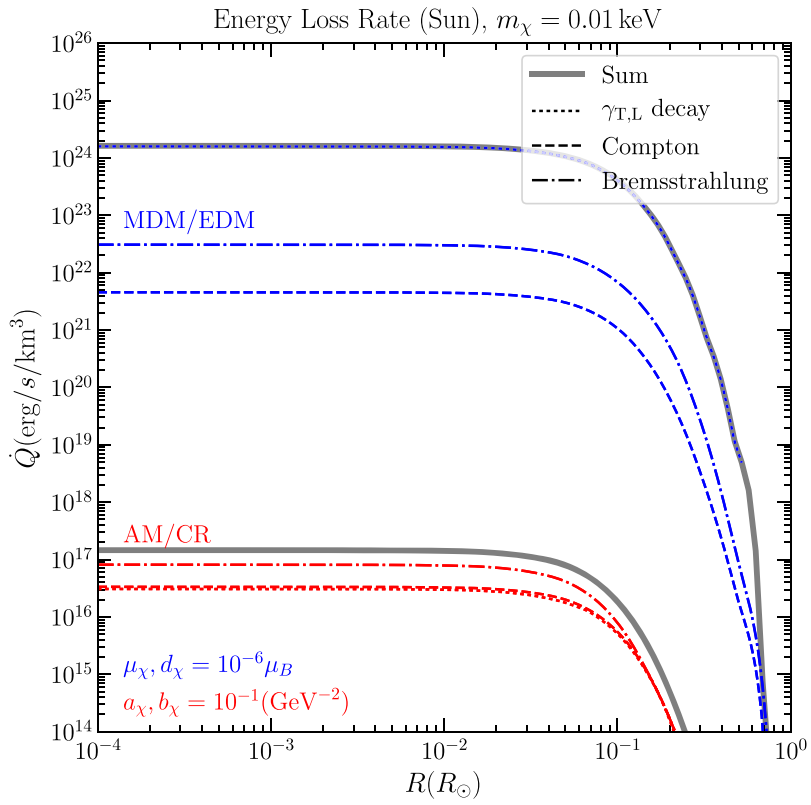

FIG. 3. Left: Energy loss rates as a function of fractional stellar radius from $\gamma_{\mathrm{T}, \mathrm{L}}$ decay (dotted lines), Compton production (dashed lines) and electron bremsstrahlung (dash-dotted lines) for $m_{\chi}=0.01 \mathrm{keV}$ and $\mu_{\chi}\left(\right.$ or $\left.d_{\chi}\right)=10^{-6} \mu_{B}$ and $a_{\chi}\left(\right.$ or $\left.b_{\chi}\right)=0.1 / \mathrm{GeV}^{2}$ in the representative HB star we consider. The sum of all processes is shown by the thick gray line, which for MDM/EDM interactions practically coincides with plasmon decay. Right: The same processes as in the left panel but for the Sun. 

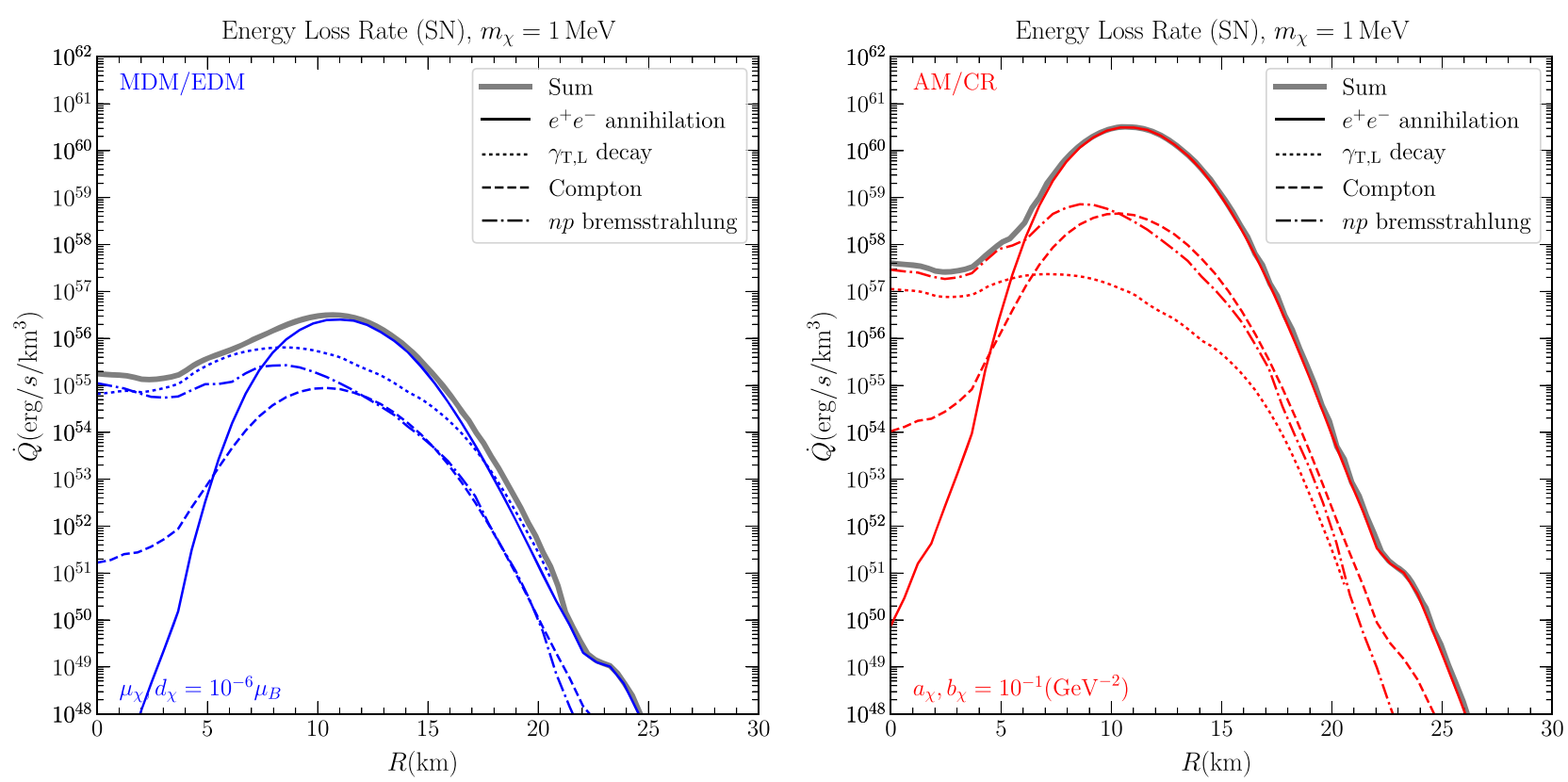

FIG. 4. Left: Energy loss rates inside PNS for MDM/EDM interactions with $\mu_{\chi}\left(\right.$ or $\left.d_{\chi}\right)=10^{-6} \mu_{B}$ and $m_{\chi}=1 \mathrm{MeV}$ are shown for all computed processes, namely, $e^{+} e^{-}$annihilation (thin solid line), $\gamma_{\mathrm{T}, \mathrm{L}}$ photon decay (dotted line), Compton production (dashed line) and $n p$ bremsstrahlung (dash-dotted line). The sum of all contributions is the thick solid line. Right: The same processes as in the left panel but for $\mathrm{AM} / \mathrm{CR}$ interactions with $a_{\chi}\left(\right.$ or $\left.b_{\chi}\right)=0.1 / \mathrm{GeV}^{2}$.

close to kinematic end points. As can be seen, for dimension5 operators the decay process (dotted lines) dominates over bremsstrahlung (dash-dotted lines) and Compton scattering (dashed lines) processes in both $\mathrm{HB}$ and the Sun. For dimension- 6 operators, the contribution of Compton scattering is comparable to that of decay processes in HB while in the Sun all three processes are of comparable importance.

Applying the criteria for the maximum allowable energy loss of Sec. III, we obtain the excluded shaded regions in Figs. 5 and 6 as labeled. The strongest limits are provided by RG stars. They have a higher core temperature, $T=8.6 \mathrm{keV}$, compared to HB stars or the Sun, favoring an emission process that is UV-biased because of the considered higher-dimensional operators. In the low mass region, for $2 m_{\chi}<\omega_{p}$, the limits are governed by $\gamma_{\mathrm{T}, \mathrm{L}}$ decays, and become independent of $\chi$ mass quickly. Once the decay process is kinematically forbidden, the limits become determined by the bremsstrahlung and Compton scattering processes. As can be seen, the critical values of $m_{\chi}$ where this happens for RG, HB, and the Sun are reflective of the differing core-plasma frequencies (A3) of the respective systems. Furthermore, the mass-dimension 5 constraints on MDM and EDM are practically identical; differences only appear in the kinematic endpoint region.

\section{B. Limits from SN1987A}

Limits on $\chi$-photon interactions from SN1987A have previously been estimated in our earlier paper [30], largely following the approach of [56], and considering $e^{+} e^{-}$ annihilation but with plasmon decay neglected. Here we revisit these constraints in light of a more detailed calculation. Dark state pairs with mass $m_{\chi} \lesssim 400 \mathrm{MeV}$ can be efficiently produced inside PNS, predominantly through $e^{+} e^{-}$annihilation as positrons are thermally supported. Nevertheless, we will consider all processes in Fig. 1 except for electron bremsstrahlung as it is significantly weaker than the others; see Fig. 4 for one example with $m_{\chi}=1 \mathrm{MeV}$.

When the particles stream freely after production and are hence able to escape from the PNS core, the limit (10) applies. We set the size of the PNS core to be $r_{\text {core }}=15 \mathrm{~km}$ and model the PNS from which $\chi \bar{\chi}$ pairs are emitted using the simulation results of a $18 M_{\odot}$ progenitor in [64] (see Fig. 7 in Appendix A). Notice that such simulation results are based on an artificial neutrino-driven explosion method and should be taken with a grain of salt. We adopt the total energy density $\rho(r)$, temperature $T(r)$ and electron abundance $Y_{e}(r)$ profiles at $1 s$ after the core bounce. The number density of baryons can be computed as $n_{b}(r) \simeq \rho(r) / m_{p}$ and the number density of electrons can be written as $n_{e}(r) \simeq n_{b}(r) Y_{e}(r)$. Other quantities such as chemical potential of electrons $\mu_{e}(r)$, plasma frequency $\omega_{p}(r)$ and effective electron mass $m_{e}^{\text {eff }}(r)$ are derived from $n_{e}(r)$ and $T(r) . m_{e}^{\text {eff }}(r)$ is recursively solved at each radius using Eq. (A20); see Appendix A for details. In the calculations that relate to the anomalous emission, $m_{e}$ is understood to be $m_{e}^{\text {eff }}$.

The result is shown by the lower boundary of the region labeled SN1987A in Figs. 5 and 6. Compared to our previous result in [30] where only $e^{+} e^{-}$annihilation was 

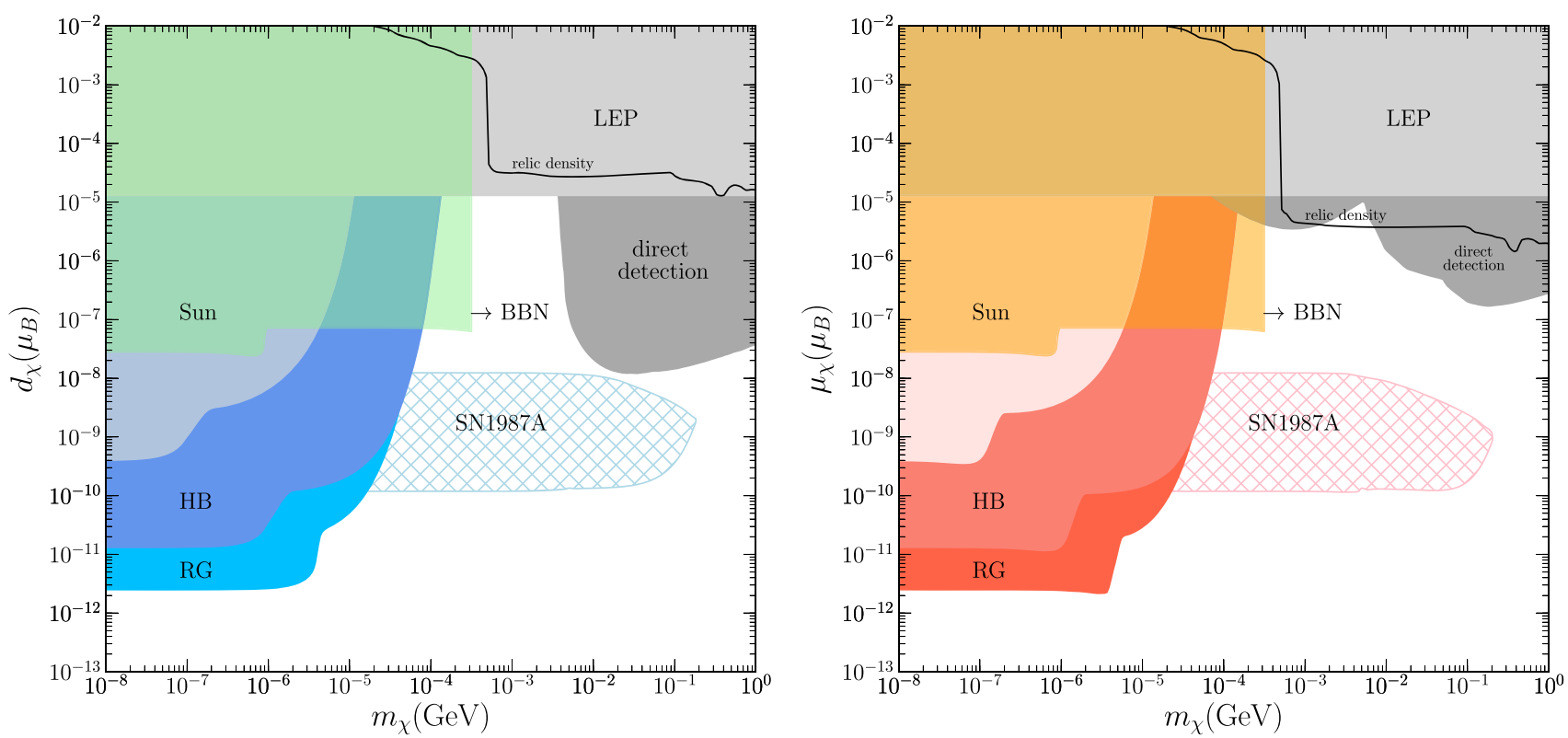

FIG. 5. Summary of constraints on the EM form factors for dim-5 operators, i.e., EDM (left) and MDM (right). Colored exclusions are derived in this work. Direct detection (only applying to dark matter) and LEP bounds are taken from our previous work [30]. On the solid black line the thermal freeze-out abundance matches the DM density.
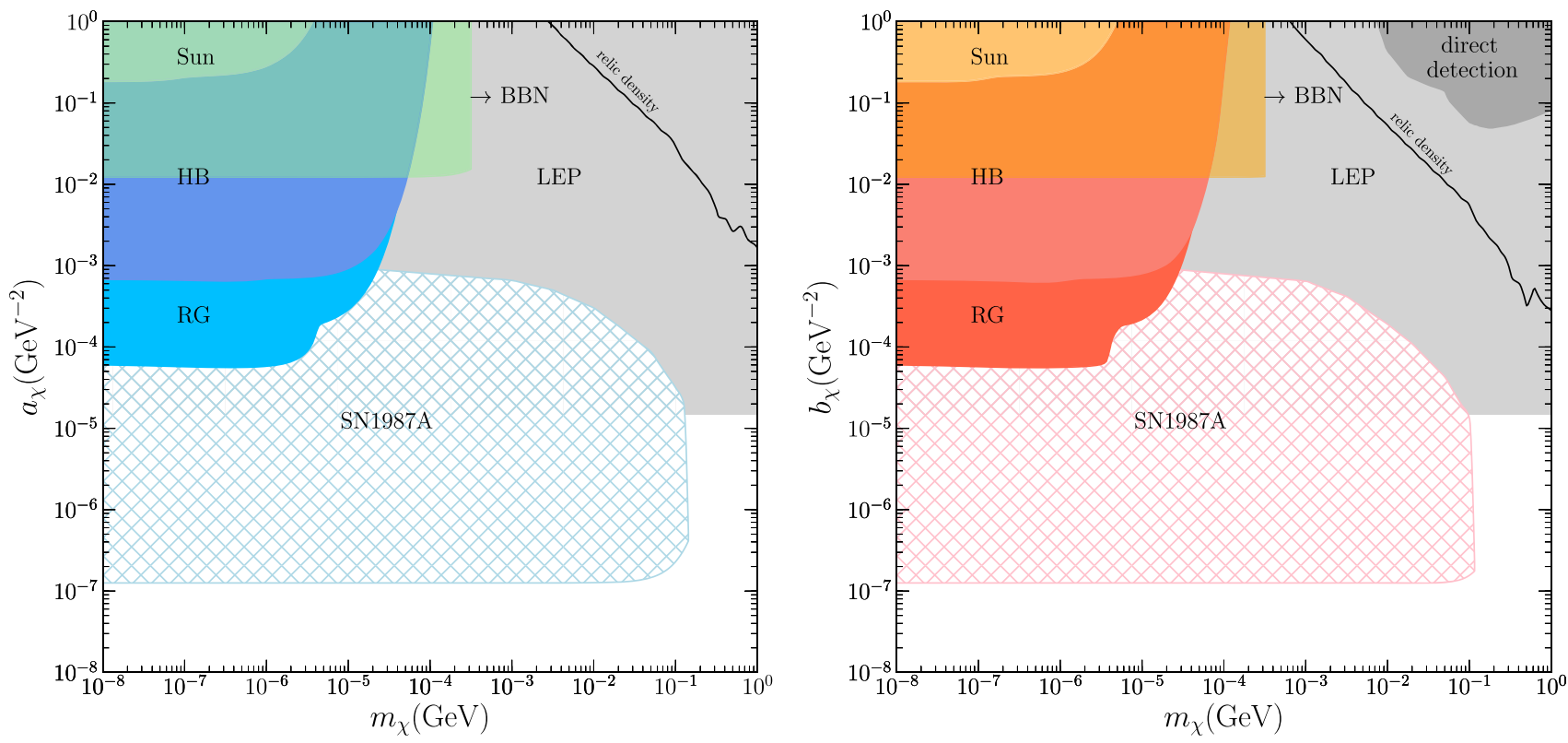

FIG. 6. Summary of constraints on the EM form factors for dim-6 operators, i.e., AM (left) and CR (right); labels are the same as in Fig. 5.

taken into account, the constraint for MDM and EDM is improved. This is traced back to the fact that the energy loss rate of $\gamma_{\mathrm{T}, \mathrm{L}}$, nucleon-bremsstrahlung and Compton scattering for MDM and EDM are comparable to $e^{+} e^{-}$annihilation into a $\chi \bar{\chi}$ pair. For $\mathrm{AM}$ and $\mathrm{CR}$, however, the results from [30] remain largely unchanged, as $\gamma_{T, L}$ decay, nucleon-bremsstrahlung and Compton scattering are less efficient.
Once the effective coupling becomes large enough, the produced $\chi$ particles will eventually come into thermal equilibrium with SM particles. Here we follow $[65,66]$ to divide the radial region into an inner "energy sphere" of radius $r_{\mathrm{ES}}$, within which light dark particles are thermalized, and an outer diffusion zone, where the $\chi$ luminosity gets attenuated by a transmission coefficient $S_{\mathrm{ES}}$. Both quantities are obtained from the energy-exchange and 

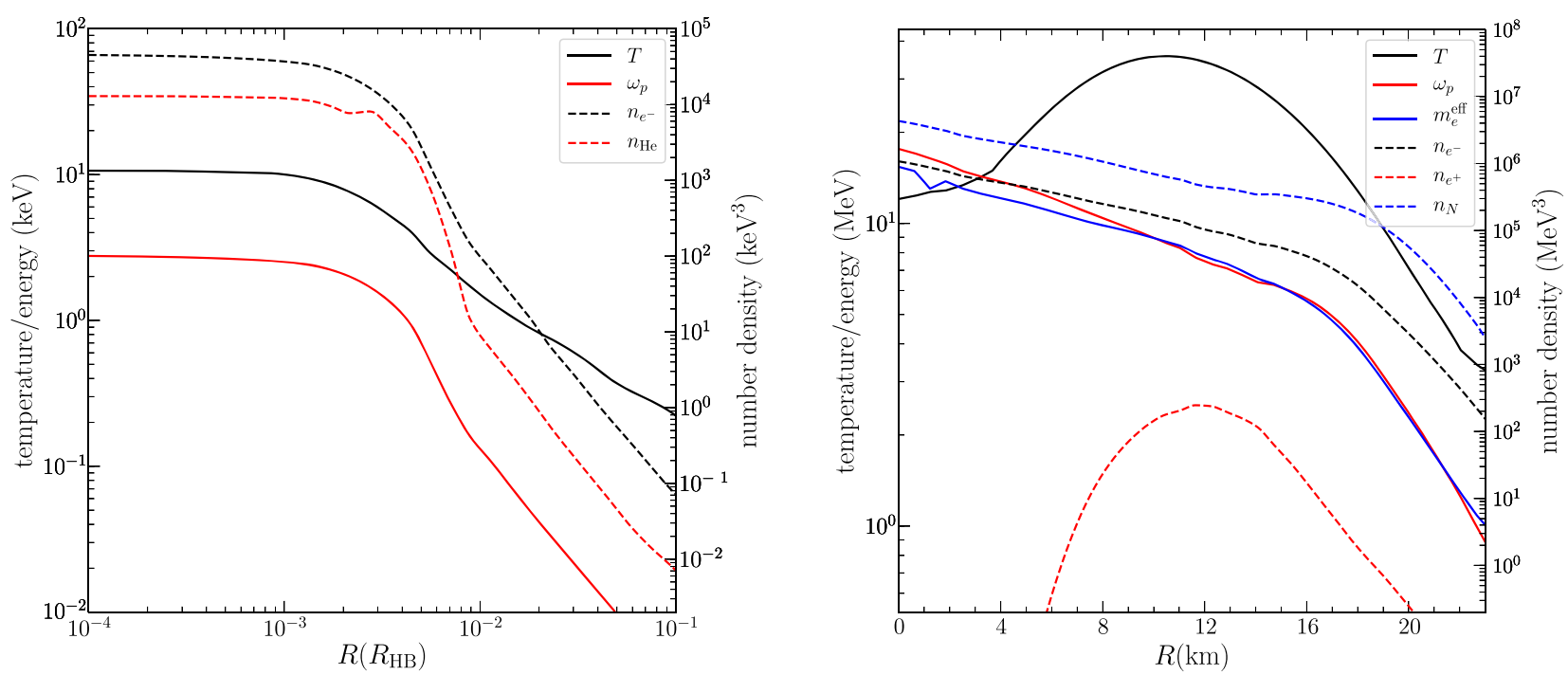

FIG. 7. Reproduced profiles of a representative $0.8 M_{\odot}$ HB star [62] (left) and of a PNS of a $18 M_{\odot}$ progenitor [64] (right) that are adopted in our work. In each panel, the left vertical axis corresponds to the values of temperature and plasma frequency (solid lines) at each radius, in units of $\mathrm{keV}(\mathrm{HB})$ or $\mathrm{MeV}(\mathrm{PNS})$, and the right vertical axis gives the number densities (dashed lines) of each particle species, in $\mathrm{keV}^{3}(\mathrm{HB})$ or $\mathrm{MeV}^{3}(\mathrm{PNS})$. For SN, the effective electron mass $m_{e}^{\text {eff }}$ is also displayed.

momentum-exchange mean-free-paths, i.e., $\lambda_{E}(r)$ and $\lambda_{M}(r)$, of the $\chi$ particle in the medium [65],

$$
\int_{r_{\mathrm{ES}}}^{r_{\mathrm{inf}}} \frac{d r}{\sqrt{\lambda_{E}(r) \lambda_{M}(r)}}=\frac{2}{3}
$$

and

$$
S_{\mathrm{ES}}=\frac{1}{1+\frac{3}{4} \int_{r_{\mathrm{ES}}}^{r_{\mathrm{inf}}} \frac{d r}{\lambda_{M}(r)}},
$$

where $r_{\text {inf }}$ is set to be $35 \mathrm{~km}$, beyond which $\chi$ particles free-stream.

In the PNS environment of interest here, the energyexchange mean-free-path is mostly governed by $e-\chi$ elastic scattering. We estimate it at each radius $r$ through

$$
\lambda_{E}(r)=\frac{\left\langle n_{\chi} v_{\chi}\right\rangle}{\left\langle n_{e} n_{\chi} \int d \cos \theta \frac{d \sigma_{e \chi}}{d \cos \theta} v_{M} \frac{|\Delta E|}{T}\right\rangle} .
$$

Here, $\langle\ldots\rangle$ denotes a thermal average over all participating particles; $n_{e}$ and $n_{\chi}$ are the number densities of electrons and $\chi$ particles which are all assumed to be in equilibrium. $d \sigma_{e \chi} / d \cos \theta$ is the differential cross section of $e-\chi$ elastic scattering. The energy-exchange in the scattering, $|\Delta E|$, is a function of the scattering angle $\theta$ in the rest frame of the medium. Although not explicitly written in the expression, we take the Pauli blocking factor for the final state electron into account.

Meanwhile, the momentum-exchange mean-free-path $\lambda_{M}(r)$ turns out to be dominated by $\chi$-nucleon scattering,
$\lambda_{M}(r)=\frac{\left\langle n_{\chi} v_{\chi}\right\rangle}{\sum_{i=n, p}\left\langle n_{i} n_{\chi} \int d \cos \theta \frac{d \sigma_{i \chi}}{d \cos \theta} v_{M}(1-\cos \theta)\right\rangle}$,

where $n_{i}$ is the number density of neutrons (n) and protons (p), provided by the PNS model [64]. The differential scattering cross section with $\chi$ is denoted as $d \sigma_{i \chi} / d \cos \theta$. In obtaining our numerical results, we have neglected the nucleon velocities in the medium frame for simplicity.

As we assume that $\chi$ stay in chemical equilibrium at $r_{\mathrm{ES}}$, its blackbody luminosity $L_{\chi}\left(r_{\mathrm{ES}}\right)$ is described by

$$
L_{\chi}\left(r_{\mathrm{ES}}\right)=\frac{g_{\chi} r_{\mathrm{ES}}^{2}}{2 \pi} \int d\left|\vec{p}_{\chi}\right| \frac{\left|\vec{p}_{\chi}\right|^{3}}{e^{\sqrt{\left|\vec{p}_{\chi}\right|^{2}+m_{\chi}^{2}} / T\left(r_{\mathrm{ES}}\right)}+1},
$$

where $g_{\chi}=4$. These $\chi$ particles are emitted towards the exterior from the energy sphere, but they continue to scatter elastically with the medium inside the diffusion zone. The $\chi$ flux-attenuation during its propagation from $r_{\mathrm{ES}}$ to $r_{\text {inf }}$ can be estimated via

$$
L_{\mathrm{inf}}=L_{\chi}\left(r_{\mathrm{ES}}\right) S_{\mathrm{ES}}
$$

in the diffusion limit [65]. That is, such attenuation only relies on the momentum-transfer cross section. Requiring $L_{\text {inf }} \leq L_{\nu}$ then leads to upper boundaries of $\mathrm{SN}$ exclusion limits shown in Figs. 5 and 6. We note in passing, that the location of the upper boundaries is still conservative, as additional "freeze-in" $\chi$-production for $r>r_{\mathrm{ES}}$ has been neglected. With respect to our previous work [30], where 
the transmission ratio $S_{\mathrm{ES}}$ was in practice taken as a simple unit-step function, the upper limits are improved by up to a factor of 2 .

\section{Related works}

Stellar bounds on the EM properties of light dark states have been studied in the literature, mostly in the context of EM properties of eV-scale (SM) neutrinos; see $[67,68]$ and references therein. In these studies, the mass of neutrino is essentially zero. Therefore, in the limit $m_{\chi} \rightarrow 0$ our results can be compared with previously derived constraints on neutrino EM interactions.

For instance, based on similar energy loss arguments, bounds on the neutrino MDM have been obtained by calculating the plasmon decay process, from RG as $\mu_{\nu} \leq$ $(2-4) \times 10^{-12} \mu_{B}$ [69-71], from HB as $\mu_{\nu} \leq(1-3) \times$ $10^{-11} \mu_{B}$ [72,73], from the Sun as $\mu_{\nu} \leq 4 \times 10^{-10} \mu_{B}$ [74]. Indeed, all these bounds are in essential agreements with our newly derived ones once the limit $m_{\chi} \rightarrow 0$ is taken.

For higher-dimensional operators, [75] estimated the anomalous energy loss rate in PNS through electron pair annihilation into light right-handed neutrinos, limiting its charge radius to be below $3.7 \times 10^{-34} \mathrm{~cm}^{2}$, that is $9.5 \times 10^{-7} \mathrm{GeV}^{-2}$, about 7 times weaker than the one presented above. This is partially due to the fact that [75] assumed an 1 order of magnitude larger luminosity as the maximum permissible energy loss.

\section{Cosmological constraints}

Light dark states may lead to extra radiation in the early Universe, and thus its coupling to the SM bath is constrained by both the predictions from big bang nucleosynthesis (BBN) and the observed cosmic microwave background (CMB). On the one hand, for the mass region considered here the $\mathrm{CMB}$ bounds depend on how it annihilates/decays. On the other hand, primordial abundance measurements of $\mathrm{D}$ and ${ }^{4} \mathrm{He}$ suggest that extra relativistic d.o.f. need to be less than that of one chiral fermion during the nucleosynthesis (see e.g., [76-78]). Thus here we require that the Dirac fermion $\chi$ is thermally diminished at $T \sim 100 \mathrm{keV}$, either due to a feeble EM formfactor coupling or by a Boltzmann-suppression induced by its mass.

The relevant bounds are also given in Figs. 5 and 6. They only constrain the parameter region with $m_{\chi} \ll 1 \mathrm{MeV}$. In the same figures, we also show the line which corresponds to the thermal freeze-out scenario which generates the observed dark matter abundance, although such scenario has been excluded by various constraints for this model; see our previous work [30]. The dominant annihilation channel is into two photons at $m_{\chi}<m_{e}$ and into a pair of electrons at $m_{\chi} \geq m_{e}$, which explains the sharp decrease of the relic density curve at $m_{\chi} \sim m_{e}$ seen in Fig. 5.

\section{CONCLUSIONS}

In this paper we explore the sensitivity of stellar systems to neutral dark states that share higher-dimensional interactions with the SM photon. To this end we choose a Dirac fermion $\chi$ that is coupled to mass dimension 5 MDM and EDM operators with respective dimensionful coefficients $\mu_{\chi}$ and $d_{\chi}$ and mass dimension 6 AM and CR operators with respective coefficients $a_{\chi}$ and $b_{\chi}$. We consider anomalous energy losses from the interior of RG and HB stars, of the Sun, and of the PNS core of SN1987A. Together with previously derived direct, indirect, and cosmological limits by us in [30], this work adds astrophysical constraints to draw a first comprehensive overview of light dark states with masses (well) below the GeV-scale and EM moment interactions.

The thermal environments of stellar interiors significantly affect (or enable) production processes of $\chi \bar{\chi}$ pairs. Before breaking it down to individual contributions, we establish the exact formula, Eq. (13), for the pair-production rate in leading order of the dark coupling. The expression factorizes into a piece that represents the probability to produce an off shell photon $\gamma^{*}$ from a SM current, and a piece that describes the production of the $\chi \bar{\chi}$ pair from that photon. The former is proportional to the imaginary parts of the longitudinal and transverse thermal photon self energies $\operatorname{Im} \Pi_{\mathrm{L}, \mathrm{T}}$. The latter are modeldependent but otherwise universal factors that represent the choice of interaction, Eq. (14). The optical theorem then allows us to identify all major production processes by studying the contributions to $\operatorname{Im} \Pi_{\mathrm{L}, \mathrm{T}}$. The approach also allows us to clarify the role of thermal resonances in these processes, i.e., the kinematic situation when the pairproducing photon goes on shell, $k^{2}=\operatorname{Re}_{\mathrm{L}, \mathrm{T}}$. We find that resonant production is entirely captured by the decay of transverse and longitudinal thermal photons or "plasmons", $\gamma_{\mathrm{T}, \mathrm{L}} \rightarrow \chi \bar{\chi}$.

We compute the rates of $\chi$-pair production and its ensuing energy loss from plasmon decay and Compton production for all systems. In addition, we evaluate $e N$ bremsstrahlung for $\mathrm{RG}, \mathrm{HB}$ and the Sun, and $e^{+} e^{-}$ annihilation and $N N$ bremsstrahlung for SN1978A. For MDM and EDM interactions, plasmon decay dominates in HB and RG stars and in the Sun. For the interactions of increased mass dimension, $\mathrm{AM}$ and $\mathrm{CR}$, the Compton (bremsstrahlung) production dominates in $\mathrm{HB}$ and $\mathrm{RG}$ (Sun). In PNS core, $e^{+} e^{-}$annihilation dominates the anomalous energy loss for $r \gtrsim 7 \mathrm{~km}$. In the most inner region the population of positrons becomes extremely Boltzmann suppressed by a decrease in temperature, and 
plasmon decay and $n p$ bremsstrahlung take over as the most important production channels. For all processes we have taken into account all important finite-temperature effects. Furthermore, in the evaluation of rates, we explicitly avoid any double counting between plasmon decay and an on shell emitted photon in bremsstrahlung and between Compton production and an on shell exchanged $t$-channel photon in bremsstrahlung.

The rates when integrated over stellar radius then become subject to the observationally inferred limits on anomalous energy loss. The resulting restrictions on the parameter space are found in Figs. 5 and 6. In the kinematically unrestricted regime $m_{\chi} \lesssim 1 \mathrm{keV}$, the stellar limits are dominated by RG with $\mu_{\chi}, d_{\chi} \leq 2 \times 10^{-12} \mu_{B}$ and $a_{\chi}, b_{\chi} \leq 6 \times 10^{-5} \mathrm{GeV}^{-2}$. All interactions are additionally constrained from SN1987A, in the windows $10^{-10} \mu_{B} \leq \mu_{\chi}, d_{\chi} \leq 10^{-8} \mu_{B}$ and $10^{-7} \mathrm{GeV}^{-2} \leq a_{\chi}, b_{\chi} \leq$ $10^{-3} \mathrm{GeV}^{-2}$ for $m_{\chi} \lesssim 10 \mathrm{MeV}$. The $\mathrm{SN}$ constraining region is bounded from above by the trapping of $\chi$ particles, which we evaluate in some detail. The presented astrophysical constraints add to a program that we have started in [30] and that aims at charting out the experimental and observational sensitivity to effective dark state-photon interactions. The stellar constraints on anomalous energy loss derived in this work yield the most important limits on the existence of effective dark sector-photon interactions for $\chi$-particles below the $\mathrm{MeV}$-scale.

\section{ACKNOWLEDGMENTS}

The authors are supported by the New Frontiers program of the Austrian Academy of Sciences. J.-L. K and L. S. are supported by the Austrian Science Fund FWF under the Doctoral Program No. W1252-N27 Particles and Interactions. We acknowledge the use of computer packages for algebraic calculations $[79,80]$.

\section{APPENDIX A: PHOTONS IN A THERMAL MEDIUM}

The processes depicted in Fig. 1 are fundamentally affected (or enabled) by the in-medium modified photon dispersion. Here we collect the central results that go into the computation of the energy loss rates (our convention largely follows [3]). The central quantity measuring the strength of the medium-effect is the plasma frequency $\omega_{p}$, obtained through

$$
\omega_{p}^{2}=\frac{4 \alpha}{\pi} \int_{0}^{\infty} d p \frac{p^{2}}{E}\left(1-\frac{1}{3} v^{2}\right)\left(f_{e^{-}}+f_{e^{+}}\right),
$$

where $v=p / E$ is the velocity of electrons or positrons, and $f_{e^{-}}$and $f_{e^{+}}$are their respective Fermi-Dirac distributions, $f_{e^{ \pm}}=\left[e^{\left(E \pm \mu_{e}\right) / T}+1\right]^{-1}$.

Equation (A1) takes on the following analytic forms in the classical, degenerate and relativistic limit respectively:

$$
\omega_{p}^{2} \simeq \begin{cases}\frac{4 \pi \alpha n_{e}}{m_{e}}\left(1-\frac{5}{2} \frac{T}{m_{e}}\right) & \text { classical } \\ \frac{4 \pi \alpha n_{e}}{E_{F}}=\frac{4 \alpha}{3 \pi} p_{F}^{2} v_{F} & \text { degenerate } \\ \frac{4 \alpha}{3 \pi}\left(\mu_{e}^{2}+\frac{1}{3} \pi^{2} T^{2}\right) & \text { relativistic }\end{cases}
$$

where $\alpha$ is the fine-structure constant, $n_{e}$ is the number density of electrons, $p_{F}=\left(3 \pi^{2} n_{e}\right)^{1 / 3}$ is the Fermi momentum, $E_{F}=\sqrt{m_{e}^{2}+p_{F}^{2}}$ is the Fermi energy and $v_{F}=p_{F} / E_{F}$ is the Fermi velocity. Here "classical" refers to a nonrelativistic $\left(T \ll m_{e}\right)$ and nondegenerate $\left(T \gg \mu_{e}-m_{e}\right)$ plasma.

The PNS core of a $\mathrm{SN}$ is both in a relativistic and degenerate regime, and we find that the relativistic limit above yields a better fit to the general form of $\omega_{p}$ in (A1) than the degenerate limit; the latter exhibits a $10 \%$ deviation. The core of a RG star is nonrelativistic but degenerate whereas HB stars and the Sun are well described by the classical limit. In our numerical calculations, we adopt $\omega_{p}$ computed from Eq. (A1), avoiding any ambiguities of taking limiting cases. Representative values of $\omega_{p}$ at the cores of all stellar objects are summarized as

$$
\omega_{p} \sim\left\{\begin{array}{ll}
0.3 \mathrm{keV} & \text { Sun's core } \\
2.6 \mathrm{keV} & \text { HB's core } \\
8.6 \mathrm{keV} & \text { RG's core } \\
17.6 \mathrm{MeV} & \text { SN's core }
\end{array} .\right.
$$

The computation of most of the processes requires the inmedium photon propagator. Picking Coulomb gauge, for a photon carrying 4-momentum $k=(\omega, \vec{k})$, the latter divides into longitudinal (L) and transverse $(\mathrm{T})$ parts [81],

$$
\begin{aligned}
D_{00} & =\frac{k^{2}}{|\vec{k}|^{2}\left(k^{2}-\Pi_{\mathrm{L}}\right)} g_{00}, \\
D_{i j} & =\frac{1}{k^{2}-\Pi_{\mathrm{T}}}\left(\delta_{i j}-\frac{k_{i} k_{j}}{|\vec{k}|^{2}}\right),
\end{aligned}
$$

where $k_{i}$ is the Cartesian component of the photon threemomentum (magnitude $|\vec{k}|$ ). Using [81] and adopting the conventions of [3,5], the real part of the polarization functions $\Pi_{T, L}$ in the rest frame of the (isotropic) thermal bath reads

$$
\begin{aligned}
\operatorname{Re} \Pi_{\mathrm{T}} & =\frac{3 \omega^{2}}{2 v_{*}^{2}|\vec{k}|^{2}} \omega_{p}^{2}\left[1-\frac{\omega^{2}-v_{*}^{2}|\vec{k}|^{2}}{2 \omega v_{*}|\vec{k}|} \ln \frac{\omega+v_{*}|\vec{k}|}{\omega-v_{*}|\vec{k}|}\right], \\
\operatorname{Re} \Pi_{\mathrm{L}} & =3 \omega_{p}^{2}\left(\frac{\omega^{2}-|\vec{k}|^{2}}{v_{*}^{2}|\vec{k}|^{2}}\right)\left[\frac{\omega}{2 v_{*}|\vec{k}|} \ln \frac{\omega+v_{*}|\vec{k}|}{\omega-v_{*}|\vec{k}|}-1\right] .
\end{aligned}
$$

The full expressions for the dispersion relations $k^{2}-\Pi_{\mathrm{L}, \mathrm{T}}=0$ then relate the energies of an on shell photon, $\omega_{\mathrm{T}, \mathrm{L}}$, to its momentum $\vec{k}$ to order $\alpha$ [81], via 


$$
\begin{aligned}
& \omega_{\mathrm{T}}^{2}=|\vec{k}|^{2}+\omega_{p}^{2} \frac{3 \omega_{\mathrm{T}}^{2}}{2 v_{*}^{2}|\vec{k}|^{2}}\left[1-\frac{\omega_{\mathrm{T}}^{2}-v_{*}^{2}|\vec{k}|^{2}}{2 \omega_{\mathrm{T}} v_{*}|\vec{k}|} \ln \frac{\omega_{\mathrm{T}}+v_{*}|\vec{k}|}{\omega_{\mathrm{T}}-v_{*}|\vec{k}|}\right], \\
& \omega_{\mathrm{L}}^{2}=\omega_{p}^{2} \frac{3 \omega_{\mathrm{L}}^{2}}{v_{*}^{2}|\vec{k}|^{2}}\left[\frac{\omega_{\mathrm{L}}}{2 v_{*}|\vec{k}|} \ln \frac{\omega_{\mathrm{L}}+v_{*}|\vec{k}|}{\omega_{\mathrm{L}}-v_{*}|\vec{k}|}-1\right] .
\end{aligned}
$$

Equations (A6) are also valid to order $|\vec{k}|^{2}$ at small $|\vec{k}|$ for all temperatures and electron number densities. Throughout the paper, we always use $\omega_{\mathrm{T}, \mathrm{L}}$, as functions of $|\vec{k}|$, to denote the energy of an on shell thermal photon, which satisfies Eqs. (A6), and use $\omega$ for off shell photons.

Longitudinal photons are populated up to a wave number $k_{\max }$, beyond which the longitudinal dispersion relation crosses the light cone and L-modes become damped, with

$$
k_{\max }=\left[\frac{3}{v_{*}^{2}}\left(\frac{1}{2 v_{*}} \ln \frac{1+v_{*}}{1-v_{*}}-1\right)\right]^{1 / 2} \omega_{p},
$$

and in the relativistic limit $k_{\max } \rightarrow \infty$. In these equations, the mobility of charges is captured by the typical velocity of electrons, $v_{*} \equiv \omega_{1} / \omega_{p}$, where

$$
\omega_{1}^{2}=\frac{4 \alpha}{\pi} \int_{0}^{\infty} d p \frac{p^{2}}{E}\left(\frac{5}{3} v^{2}-v^{4}\right)\left(f_{e^{-}}+f_{e^{+}}\right) .
$$

In the three limits mentioned previously, $v_{*}$ can be approximated as

$$
v_{*} \simeq \begin{cases}\sqrt{5 T / m_{e}} & \text { classical } \\ v_{F} & \text { degenerate } \\ 1 & \text { relativistic }\end{cases}
$$

Finally, as alluded to in the main text, the processes we consider are nonresonant in the photon exchange and $\operatorname{Im} \Pi_{T, L}$ can be neglected throughout.

In turn, the computation of in-medium photon decay, i.e., the process (1a), requires the description of external inmedium photon states. For propagation in the $z$-direction, i.e., $k_{x}=k_{y}=0$, the transverse and longitudinal polarization vectors are given by

$$
\epsilon_{\mathrm{T}}^{\mu}=(0,1(0), 0(1), 0), \quad \epsilon_{\mathrm{L}}^{\mu}=\frac{1}{\sqrt{\omega_{L}^{2}-|\vec{k}|^{2}}}\left(|\vec{k}|, 0,0, \omega_{L}\right) .
$$

In all cases $\epsilon^{\mu} \epsilon_{\mu}=-1$ and $\epsilon^{\mu} k_{\mu}=0$.

Furthermore, the in-medium coupling of the photon to the EM current is modified by the vertex renormalization constants $Z_{\mathrm{T}, \mathrm{L}} \equiv\left(1-\partial \Pi_{\mathrm{T}, \mathrm{L}} / \partial \omega_{\mathrm{T}, \mathrm{L}}^{2}\right)^{-1}$. For the convention adopted here, they are equivalent to the ones given in [3]
$Z_{\mathrm{T}}=\frac{2 \omega_{\mathrm{T}}^{2}\left(\omega_{\mathrm{T}}^{2}-v_{*}^{2}|\vec{k}|^{2}\right)}{3 \omega_{p}^{2} \omega_{\mathrm{T}}^{2}+\left(\omega_{\mathrm{T}}^{2}+|\vec{k}|^{2}\right)\left(\omega_{\mathrm{T}}^{2}-v_{*}^{2}|\vec{k}|^{2}\right)-2 \omega_{\mathrm{T}}^{2}\left(\omega_{\mathrm{T}}^{2}-|\vec{k}|^{2}\right)}$,

$Z_{\mathrm{L}}=\frac{2\left(\omega_{\mathrm{L}}^{2}-v_{*}^{2}|\vec{k}|^{2}\right)}{3 \omega_{p}^{2}-\left(\omega_{\mathrm{L}}^{2}-v_{*}^{2}|\vec{k}|^{2}\right)} \frac{\omega_{L}^{2}}{\omega_{L}^{2}-|\vec{k}|^{2}}$.

These factors are attached to each zero-temperature vertex factor involving an external photon state. For internal photons, this effect is already accounted for in the momentum-dependent self-energy $\Pi_{\mathrm{T}, \mathrm{L}}(\omega, \vec{k})$.

For thermal corrections to the electron mass which is relevant for PNS, we closely follow [82]. For an electron with a general 4-momentum $p=(E, \vec{p})$ in a neutral medium where the positron number density is negligible, we first introduce the four functions below,

$$
\begin{aligned}
& A_{e}=\frac{-\alpha}{4 \pi|\vec{p}|} \int_{0}^{\infty} d q \frac{q f_{e^{-}}(q)}{\sqrt{q^{2}+m_{e}^{2}}}\left[4|\vec{p}| q-\left(p^{2}+m_{e}^{2}\right) L_{2}\right], \\
& C_{e}=\frac{\alpha m_{e}}{\pi|\vec{p}|} \int_{0}^{\infty} d q \frac{q f_{e^{-}}(q)}{\sqrt{q^{2}+m_{e}^{2}}}\left(-L_{2}\right), \\
& A_{\gamma}=\frac{-\alpha}{4 \pi|\vec{p}|} \int_{0}^{\infty} d q f_{\gamma}(q)\left[8|\vec{p}| q+\left(p^{2}+m_{e}^{2}\right)\left(L_{3}-L_{4}\right)\right],
\end{aligned}
$$

$C_{\gamma}=\frac{\alpha m_{e}}{\pi|\vec{p}|} \int_{0}^{\infty} d q f_{\gamma}(q)\left(L_{3}-L_{4}\right)$

where $q$ here is the absolute value of the 3-momentum of medium particles (electron, photon) that is integrated over and $L_{1,2,3,4}$ are functions of $q$ in terms of

$$
\begin{aligned}
& L_{1}(q)=\ln \left[\frac{2\left(E \sqrt{q^{2}+m_{e}^{2}}+|\vec{p}| q\right)-p^{2}-m_{e}^{2}}{2\left(E \sqrt{q^{2}+m_{e}^{2}}-|\vec{p}| q\right)-p^{2}-m_{e}^{2}}\right], \\
& L_{2}(q)=\ln \left[\frac{2\left(E \sqrt{q^{2}+m_{e}^{2}}+|\vec{p}| q\right)+p^{2}+m_{e}^{2}}{2\left(E \sqrt{q^{2}+m_{e}^{2}}-|\vec{p}| q\right)+p^{2}+m_{e}^{2}}\right], \\
& L_{3}(q)=\ln \left[\frac{2(E q+|\vec{p}| q)+p^{2}-m_{e}^{2}}{2(E q-|\vec{p}| q)+p^{2}-m_{e}^{2}}\right], \\
& L_{4}(q)=\ln \left[\frac{2(E q+|\vec{p}| q)-p^{2}+m_{e}^{2}}{2(E q-|\vec{p}| q)-p^{2}+m_{e}^{2}}\right] .
\end{aligned}
$$

Here $m_{e}$ is the zero-temperature mass of electron, $0.511 \mathrm{MeV}$, while $f_{\gamma}(p)$ and $f_{e^{-}}(p)$ give the thermal momentum distribution functions of photon and electron (per d.o.f.). We have set $f_{e^{+}}(p)=0$ in the above equations. In the end, we take the approximation made in [83] to obtain that 
$m_{e}^{\mathrm{eff}}(p)=\sqrt{m_{e}^{2}-2\left(A_{\gamma}+A_{e}\right)-2 m_{e}\left(C_{\gamma}+C_{e}\right)}$.

We have neglected thermal corrections to $\chi$ states. In the phenomenologically relevant regime, their coupling to the thermal bath is very weak.

Finally, we have reproduced the profiles of the HB model from [62], and PNS model from [64], adopted in this work, as shown in Fig. 7, where neutrality and $\mu_{e^{-}}+\mu_{e^{+}}=0$ at each radius have been taken for granted for the PNS profile.

\section{APPENDIX B: DECAY RATE AND CROSS SECTION CALCULATIONS}

In this Appendix we collect some further details that enter the calculation of the $\chi \bar{\chi}$ production cross sections found in Sec. IV.

\section{Full expression of $\chi$-pair production rate}

For any process that produces a $\chi \bar{\chi}$ pair through a photon propagator of 4-momentum $k=(\omega, \vec{k})$, its spin-summed squared matrix element can be written in terms of

$$
\sum_{\text {spins }}|\mathcal{M}|^{2}=D_{\mu \nu}(k) D_{\rho \sigma}^{*}(k) \mathcal{T}_{\mathrm{SM}}^{\mu \rho} \mathcal{T}_{\chi}^{\nu \sigma},
$$

where the in-medium photon propagator $D^{\mu \nu}$ is given by (A4), while $\mathcal{T}_{\mathrm{SM}}^{\mu \rho}$ and $\mathcal{T}_{\chi}^{\nu \sigma}$ represent the corresponding squared matrix elements of the SM current, i.e., $\mathrm{SM} \rightarrow \gamma^{*}(k)\left(+\mathrm{SM}^{\prime}\right)$, and the dark current, i.e., $\gamma^{*}(k) \rightarrow \chi\left(p_{\chi}\right)+\bar{\chi}\left(p_{\bar{\chi}}\right)$, of which the latter is given by

$$
\mathcal{T}_{\chi}^{\nu \sigma}=\operatorname{Tr}\left[\left(\not \chi_{\chi}+m_{\chi}\right) \Gamma^{\nu}(k)\left(\not \bar{\chi}_{\bar{\chi}}-m_{\chi}\right) \Gamma^{\sigma}(-k)\right] .
$$

The vertex factors $\Gamma^{\nu}(k)$ are derived from the Lagrangians (5) and (6) through the usual prescription of obtaining Feynman rules; see [30] for the explicit expressions. Generalizing Eq. (5.156) of [53] yields an expression for the exact $\chi \bar{\chi}$ differential production rate per volume,

$$
\frac{d \dot{N}_{\chi}}{d^{4} k}=\frac{1}{(2 \pi)^{4}} D_{\mu \nu}(k) D_{\rho \sigma}^{*}(k)\left(\frac{2 \operatorname{Im} \Pi^{\mu \rho}(k)}{e^{\omega / T}-1}\right) I_{\chi}^{\nu \sigma},
$$

where $\operatorname{Im} \Pi^{\mu \rho}$ is the imaginary part of the thermal photon self-energy induced by all possible SM currents. In the medium it is decomposed into longitudinal and transverse components, $\operatorname{Im} \Pi_{L, T}$, as shown in Eq. (12) in the main text. The factor $I^{\nu \sigma}$ is the 2-body final state integrated over its phase space,

$$
I_{\chi}^{\nu \sigma}=\int d \Pi_{i=\chi, \bar{\chi}}(2 \pi)^{4} \delta^{4}\left(k-p_{\chi}-p_{\bar{\chi}}\right) \mathcal{T}_{\chi}^{\nu \sigma},
$$

where $d \Pi_{i}=\prod_{i} d^{3} \vec{p}_{i}(2 \pi)^{-3}\left(2 E_{i}\right)^{-1}$, as mentioned in the main text. The integration can be executed in an arbitrary frame, and in particular in the rest frame of the thermal bath by adopting Lenard's formula [84], generalized to massive final states. We find

$$
\begin{aligned}
& \int d \Pi_{i=\chi, \bar{\chi}}(2 \pi)^{4} \delta^{4}\left(k-p_{\chi}-p_{\bar{\chi}}\right) p_{\chi}^{\mu} p_{\bar{\chi}}^{\nu} \\
& =\frac{1}{96 \pi}\left(A k^{2} g^{\mu \nu}+2 B k^{\mu} k^{\nu}\right),
\end{aligned}
$$

where the coefficients $A$ and $B$ are given by

$$
A=\left(1-\frac{4 m_{\chi}^{2}}{s_{\chi \bar{\chi}}}\right)^{3 / 2}, \quad B=\sqrt{1-\frac{4 m_{\chi}^{2}}{s_{\chi \bar{\chi}}}}\left(1+\frac{2 m_{\chi}^{2}}{s_{\chi \bar{\chi}}}\right),
$$

with $s_{\chi \bar{\chi}}=k^{2}$. In terms of the functions $f\left(s_{\chi \bar{\chi}}\right)$ defined in (14), the factor $I^{\nu \sigma}$ is then explicitly given by

$$
I^{\nu \sigma}=\frac{1}{8 \pi} \sqrt{1-\frac{4 m_{\chi}^{2}}{s_{\chi \bar{\chi}}}} f\left(s_{\chi \bar{\chi}}\right)\left(-g^{\nu \sigma}+\frac{k^{\nu} k^{\sigma}}{s_{\chi \bar{\chi}}}\right) .
$$

Putting all of the above together, we obtain the differential production rate per volume (13) found in the main text which we repeat here for convenience,

$$
\begin{aligned}
\frac{d \dot{N}_{\chi}}{d^{4} k}= & \frac{1}{64 \pi^{5}}\left[-\frac{\operatorname{Im} \Pi_{\mathrm{L}}(k)}{\left|s_{\chi \bar{\chi}}-\Pi_{\mathrm{L}}\right|^{2}}-\frac{2 \operatorname{Im} \Pi_{\mathrm{T}}(k)}{\left|s_{\chi \bar{\chi}}-\Pi_{\mathrm{T}}\right|^{2}}\right] \\
& \times f_{B}(\omega) f\left(s_{\chi \bar{\chi}}\right) \sqrt{1-\frac{4 m_{\chi}^{2}}{s_{\chi \bar{\chi}}}}
\end{aligned}
$$

where $f_{B}(\omega)=\left(e^{\omega / T}-1\right)^{-1}$ is the Bose-Einstein momentum distribution of thermal bosons. During the derivation we have used that

$$
-g^{\nu \sigma}+\frac{k^{\nu} k^{\sigma}}{s_{\chi \bar{\chi}}}=\epsilon_{\mathrm{T}, 1}^{\nu} \epsilon_{\mathrm{T}, 1}^{\sigma}+\epsilon_{\mathrm{T}, 2}^{\nu} \epsilon_{\mathrm{T}, 2}^{\sigma}+\epsilon_{\mathrm{L}}^{\nu} \epsilon_{\mathrm{L}}^{\sigma} .
$$

\section{Leading contributions to $\operatorname{Im} \Pi_{T, L}$}

We now demonstrate that (13) or, equivalently, (B3) contain the leading production mechanisms considered in this paper. In particular we clarify the role of resonances, and that they are accounted for by the process $\gamma_{\mathrm{T}, \mathrm{L}} \rightarrow \chi \bar{\chi}$. To this end, we isolate the pole contribution to the total production rate, i.e., the case $s_{\chi \bar{\chi}}=\operatorname{Re} \Pi_{\mathrm{L}, \mathrm{T}}$. To this end, we adopt the narrow width approximation,

$$
\lim _{\operatorname{Im} \Pi_{\mathrm{L}, \mathrm{T}} \rightarrow 0} \frac{-\operatorname{Im} \Pi_{\mathrm{L}, \mathrm{T}}(k)}{\pi\left|s_{\chi \bar{\chi}}-\Pi_{\mathrm{L}, \mathrm{T}}\right|^{2}}=\delta\left(s_{\chi \bar{\chi}}-\operatorname{Re} \Pi_{\mathrm{L}, \mathrm{T}}\right),
$$

where $\operatorname{Im} \Pi_{\mathrm{L}, \mathrm{T}}<0$. Then noting that $\operatorname{Re} \Pi_{\mathrm{L}, \mathrm{T}}$ is also a function of $s_{\chi \bar{\chi}}$ and writing $d^{4} k=d^{3} \vec{k} d s_{\chi \bar{\chi}} /(2 \omega)$ yield 


$$
\begin{aligned}
\dot{N}_{\chi}^{\mathrm{T}, \mathrm{L}}= & g_{\mathrm{T}, \mathrm{L}} \int \frac{d^{3} \vec{k}}{(2 \pi)^{3}} f_{B}\left(\omega_{\mathrm{T}, \mathrm{L}}\right) \\
& \times\left[\frac{Z_{\mathrm{T}, \mathrm{L}} f\left(\operatorname{Re} \Pi_{\mathrm{T}, \mathrm{L}}\right)}{16 \pi \omega_{\mathrm{T}, \mathrm{L}}} \sqrt{1-\frac{4 m_{\chi}^{2}}{\operatorname{Re} \Pi_{\mathrm{T}, \mathrm{L}}}}\right],
\end{aligned}
$$

where $g_{\mathrm{T}}=2$ and $g_{\mathrm{L}}=1$, counting the d.o.f. of the photon modes. Now both $\omega_{T, L}$ and $\operatorname{Re} \Pi_{\mathrm{L}, \mathrm{T}}$ need to satisfy the photon dispersion relation with a 3 -momentum $\vec{k}$ due to the $\delta$-function above. As will be calculated below and given explicitly in (15), the term in the parentheses is exactly the decay rate of $\gamma_{\mathrm{L}, \mathrm{T}}$ into $\chi$ pairs.

In a next step we further verify that the contribution of the one electron loop (OEL) to $\operatorname{Im}_{\mu \rho}$ induces the production rate of $\chi$ from electron pair annihilation, ${ }^{5}$ the process (2b). In this case, it is easier to start with (B3), where according to the in-medium optical theorem (see Fig. 2) we may write

$$
\begin{aligned}
\left.2 \operatorname{Im} \Pi^{\mu \rho}\right|_{\mathrm{OEL}}= & \int d \Pi_{i=1,2} \mathcal{T}_{e}^{\mu \rho}\left(1-f_{e^{-}}-f_{e^{+}}\right) \\
& \times(2 \pi)^{4} \delta^{4}\left(k-p_{1}-p_{2}\right),
\end{aligned}
$$

where $\mathcal{T}_{e}^{\mu \rho}=\mathcal{M}_{\gamma^{*} \rightarrow e^{+} e^{-}}^{\mu} \mathcal{M}_{e^{+} e^{-} \rightarrow \gamma^{*}}^{\rho}$, and $f_{e^{\mp}}$ gives the momentum distribution function of $e^{-}\left(p_{1}\right), e^{+}\left(p_{2}\right)$ per d.o.f. as defined above. Moreover, terms that are kinetically forbidden for $k^{2}>0$ have been neglected [50]. The presence of $\left(1-f_{e^{-}}-f_{e^{+}}\right)$is due to quantum statistics, and would disappear for classical particles. Substituting this expression into (B3) gives

$$
\begin{aligned}
\left.\frac{d \dot{N}_{\chi}}{d^{4} k}\right|_{\mathrm{OEL}}= & \int d \Pi_{i=1,2, \chi, \bar{\chi}}\left|\mathcal{M}_{\mathrm{ann}}\right|^{2}\left(1-f_{e^{-}}-f_{e^{+}}\right) f_{B}(\omega) \\
& \times(2 \pi)^{4} \delta^{4}\left(k-p_{1}-p_{2}\right) \delta^{4}\left(k-p_{\chi}-p_{\bar{\chi}}\right) .
\end{aligned}
$$

Then for the Fermi-Dirac distribution function $f_{e^{ \pm}}$and the Bose-Einstein distribution $f_{B}(\omega)$ with the energy conservation $E_{1}+E_{2}=\omega$, there exists the relation

$$
\left(\frac{f_{e^{-}}}{1-f_{e^{-}}}\right)\left(\frac{f_{e^{+}}}{1-f_{e^{+}}}\right)=\frac{f_{B}(\omega)}{1+f_{B}(\omega)}
$$

allowing us to rewrite the number production rate per volume above as

$$
\begin{aligned}
\left.\dot{N}_{\chi}\right|_{\mathrm{OEL}}= & \int d \Pi_{i=1,2, \chi, \bar{\chi}}\left|\mathcal{M}_{\mathrm{ann}}\right|^{2} f_{e^{-}} f_{e^{+}} \\
& \times(2 \pi)^{4} \delta^{4}\left(p_{1}+p_{2}-p_{\chi}-p_{\bar{\chi}}\right),
\end{aligned}
$$

\footnotetext{
${ }^{5}$ The contribution of the two and three electron loops to $\operatorname{Im}_{\mu \rho}$ correspond to Compton scattering and bremsstrahlung, respectively.
}

after integrating over $d^{4} k$ on both sides. The last expression transforms precisely to the corresponding energy loss rate (19), once both the energy-loss factor $\left(E_{1}+E_{2}\right)$ and fermionic d.o.f. $f_{e^{ \pm}}$are taken in account.

\section{3. $\gamma_{T L}$ decay to dark states}

In the following Appendixes we calculate the leading processes in the usual Feynman-diagrammatic approach using tree-level perturbation theory augmented by the thermal corrections outlined in Appendix A. The decay of a transverse or longitudinal photon of 4-momentum $k$ to a pair of dark states $\bar{\chi}\left(p_{\bar{\chi}}\right)+\chi\left(p_{\chi}\right)$ is described by the spinsummed squared matrix element,

$$
\sum_{\text {spins }}\left|\mathcal{M}_{\mathrm{T}, \mathrm{L}}\right|^{2}=Z_{\mathrm{T}, \mathrm{L}} \epsilon_{\mu}(k) \epsilon_{\nu}^{*}(k) \mathcal{T}_{\chi}^{\mu \nu}
$$

where $Z_{\mathrm{T}, \mathrm{L}}$ is the vertex renormalization factor in (A11), $\epsilon_{\mu}$ is the photon polarization vector and $\mathcal{T}_{\chi}^{\mu \nu}$ is given in (B2). The decay rate is given by the phase-space integral,

$\Gamma_{\mathrm{T}, \mathrm{L}}=\int d \Pi_{i=\chi, \bar{\chi}}(2 \pi)^{4} \delta^{4}\left(k-p_{\chi}-p_{\bar{\chi}}\right) \frac{1}{2 \omega_{\mathrm{T}, \mathrm{L}}} \sum_{\text {spins }}\left|\mathcal{M}_{\mathrm{T}, \mathrm{L}}\right|^{2}$,

where $\omega_{\mathrm{T}, \mathrm{L}}$ is the energy of the external transverse or longitudinal photon. It is useful to employ (B5). In terms of $I^{\nu \sigma}$ defined in (B6), we can write the decay rate as

$$
\Gamma_{\mathrm{T}, \mathrm{L}}=\frac{1}{2 \omega_{\mathrm{T}, \mathrm{L}}} Z_{\mathrm{T}, \mathrm{L}} \epsilon_{\mu}(k) \epsilon_{\nu}^{*}(k) I^{\mu \nu} .
$$

The explicit expression, given by (15) in the main text, is then found by using the expressions (A10) for the polarization vectors when the initial state propagates in positive $z$-direction, i.e., for $k^{\mu}=(\omega, 0,0, k)$; note that the term proportional to $k^{\mu} k^{\nu}$ in $I^{\mu \nu}$ does not contribute due to the Ward identity.

\section{4. $e^{+} e^{-}$annihilation to dark states}

Here we consider the process $e^{-}\left(p_{1}\right)+e^{+}\left(p_{2}\right) \rightarrow$ $\chi\left(p_{\chi}\right)+\bar{\chi}\left(p_{\bar{\chi}}\right)$. By setting $p_{i}=\left(E_{i}, \vec{p}_{i}\right)$ and $k=p_{1}+p_{2}$, one can define the cross sections ${ }^{6}$ in terms of the squared matrix element for annihilation $\left|\mathcal{M}_{\mathrm{ann}}\right|^{2}$,

$$
\begin{aligned}
\sigma & =\int \frac{d \Pi_{i=\chi, \bar{\chi}}}{4 E_{1} E_{2} v_{M}}(2 \pi)^{4} \delta^{4}\left(k-p_{\chi}-p_{\bar{\chi}}\right) \frac{1}{4} \sum_{\text {spins }}\left|\mathcal{M}_{\mathrm{ann}}\right|^{2} \\
& =\frac{\pi \alpha}{4 E_{1} E_{2} v_{M}} D_{\mu \nu} D_{\rho \sigma}^{*} \mathcal{T}_{e}^{\mu \rho} I_{\chi}^{\nu \sigma},
\end{aligned}
$$

\footnotetext{
${ }^{6}$ We emphasize that this leads to a Lorentz-invariant total cross section up to the thermal mass of photons, which is convenient for the phase space integral.
} 
where $\mathcal{T}_{e}^{\mu \rho}$ reads

$$
\mathcal{T}_{e}^{\mu \rho}=-2\left(s g^{\mu \rho}-2 p_{1}^{\mu} p_{2}^{\rho}-2 p_{1}^{\rho} p_{2}^{\mu}\right),
$$

and $I_{\chi}^{\nu \sigma}$ is given in (B6); here $s=k^{2}$. Furthermore, $v_{M}$ is the Møller velocity defined as $v_{M}=F /\left(E_{1} E_{2}\right)$ and the flux factor $F$ is given by

$$
F=\left[\left(p_{1} \cdot p_{2}\right)^{2}-m_{e}^{4}\right]^{1 / 2}=\frac{1}{2} \sqrt{s\left(s-4 m_{e}^{2}\right)} .
$$

Contracting the Lorentz indices then yields

$$
\sigma=\sigma_{T}+\sigma_{L}
$$

where the interference term vanishes in the Coulomb gauge, as can also be seen from (13), and the cross section for each polarization mode is given as Eqs. (17) and (18) in the main text.

\section{5. $e^{-} N$ bremsstrahlung production of dark states}

The $2 \rightarrow 3$ amplitude squared $\left|\mathcal{M}_{2 \rightarrow 3}\right|^{2}$ can be split into three parts as

$\sum_{\text {spins }} \frac{\left|\mathcal{M}_{2 \rightarrow 3}\right|^{2}}{(4 \pi \alpha)^{2} g_{1} g_{2}}=D^{\rho \beta}(q) D^{\sigma \gamma *}(q) W_{\rho \sigma} L_{\beta \gamma}^{\mu \nu} \epsilon_{\mu}^{*}(k) \epsilon_{\nu}(k)$,

where $q=p_{2}-p_{4}$ is the momentum transfer between the initial states, $L_{\beta \gamma}^{\mu \nu}$ stands for the leptonic part, $W_{\rho \sigma}$ is the hadronic tensor and $\epsilon_{\nu}(k)$ is the polarization vector of the emitted photon of virtual mass $s_{\chi \bar{\chi}}=k^{2}$. Detailed forms for $L_{\beta \gamma}^{\mu \nu}$ and $W_{\rho \sigma}$ are given in the Appendix A of our previous work [30].

The $2 \rightarrow 3$ cross section reads

$$
\sigma_{2 \rightarrow 3}=\frac{1}{4 g_{1} g_{2} E_{1} E_{2} v_{M}} \int d \Pi_{i=3,4, k} \sum_{\text {spins }}\left|\mathcal{M}_{2 \rightarrow 3}\right|^{2},
$$

where $v_{M}$ is the Møller velocity, as defined in the main text. The phase space integrations, when written in terms of Lorentz invariants reads

$$
\begin{aligned}
\sigma_{2 \rightarrow 3}= & \frac{1}{32(2 \pi)^{4} E_{1} E_{2} v_{M}} \int d s_{4} \int d t_{1} \frac{1}{\sqrt{\lambda\left(s_{4}, m_{p}^{2}, t_{1}\right)}} \\
& \times \int d t_{2} \int d p_{1 k}\left|\frac{\partial \phi_{4}^{R 4 k}}{\partial p_{1 k}}\right| \frac{1}{g_{1} g_{2}} \sum_{\text {spins }}\left|\mathcal{M}_{2 \rightarrow 3}\right|^{2} .
\end{aligned}
$$

Here, $s=\left(p_{1}+p_{2}\right)^{2}, t_{1} \equiv\left(p_{1}-p_{3}\right)^{2}, t_{2} \equiv\left(p_{2}-p_{4}\right)^{2}=q^{2}$, $s_{4} \equiv\left(p_{4}+k\right)^{2}, p_{1 k}=p_{1} \cdot k$ and $\phi_{4}^{R 4 k}$ is the azimuthal angle between $p_{4}$ and $k$ in their center of mass frame; $\lambda\left(a^{2}, b^{2}, c^{2}\right)$ is the Källén function. The Jacobian $\left|\partial \phi_{4}^{R 4 k} / \partial p_{1 k}\right|$ transforms the variable $\phi_{4}^{R 4 k}$ to the Lorentz invariant variable $p_{1 k}$.

The integration boundary of $s_{4}$ is given by

$$
\left(m_{N}+\sqrt{s_{\chi \bar{\chi}}}\right)^{2} \leq s_{4} \leq\left(\sqrt{s}-m_{e}\right)^{2},
$$

and the boundaries of $t_{1}$ and $t_{2}$ are given by

$$
\begin{aligned}
t_{1}^{ \pm}= & 2 m_{e}^{2}-\frac{1}{2 s}\left[\left(s+m_{e}^{2}-m_{N}^{2}\right)\left(s+m_{e}^{2}-s_{4}\right)\right. \\
& \left.\mp \lambda\left(s, m_{e}^{2}, m_{N}^{2}\right)^{1 / 2} \lambda\left(s, m_{e}^{2}, s_{4}\right)^{1 / 2}\right], \\
t_{2}^{ \pm}= & 2 m_{N}^{2}-\frac{1}{2 s_{4}}\left[\left(s_{4}+m_{N}^{2}-t_{1}\right)\left(s_{4}+m_{N}^{2}-s_{\chi \bar{\chi}}\right)\right. \\
& \left.\mp \lambda\left(s_{4}, m_{N}^{2}, t_{1}\right)^{1 / 2} \lambda\left(s_{4}, m_{N}^{2}, s_{\chi \bar{\chi}}\right)^{1 / 2}\right] .
\end{aligned}
$$

The physical region for $p_{1 k}$ is expressed by $n \times n$ asymmetric and symmetric Gram determinants, $G_{n}$ and $\Delta_{n}$. It reads

$$
\begin{aligned}
p_{1 k}^{ \pm}= & \frac{\left(p_{1} \cdot p_{2}\right) G_{2}\left(p_{2}, \sqrt{t_{1}} ; \sqrt{t_{1}}, k\right)}{-\Delta_{2}\left(p_{2}, \sqrt{t_{1}}\right)} \\
& -\frac{\left(\sqrt{t_{1}} \cdot p_{1}\right) G_{2}\left(p_{2}, \sqrt{t_{1}} ; p_{2}, k\right)}{-\Delta_{2}\left(p_{2}, \sqrt{t_{1}}\right)} \\
& \pm \frac{\sqrt{\Delta_{3}\left(p_{2}, \sqrt{t_{1}}, p_{1}\right) \Delta_{3}\left(p_{2}, \sqrt{t_{1}}, k\right)}}{-\Delta_{2}\left(p_{2}, \sqrt{t_{1}}\right)},
\end{aligned}
$$

and the Jacobian $\left|\partial \phi_{4}^{R 4 k} / \partial p_{1 k}\right|$ reads

$$
\left|\frac{\partial \phi_{4}^{R 4 k}}{\partial p_{1 k}}\right|=-\frac{\sqrt{-\Delta_{2}\left(p_{2}, \sqrt{t_{1}}\right)}}{\sqrt{-\Delta_{4}\left(p_{2}, \sqrt{t_{1}}, p_{1}, k\right)}} .
$$

Putting everything together, the full $2 \rightarrow 4$ cross section is given by

$$
\sigma_{2 \rightarrow 4}=\int d s_{\chi \bar{\chi}} \sigma_{2 \rightarrow 3}\left(s_{\chi \bar{\chi}}\right) \frac{f\left(s_{\chi \bar{\chi}}\right)}{16 \pi^{2} s_{\chi \bar{\chi}}^{2}} \sqrt{1-\frac{4 m_{\chi}^{2}}{s_{\chi \bar{\chi}}}} .
$$

The integration boundaries of $s_{\chi \bar{\chi}}$ are given by

$$
4 m_{\chi}^{2} \leq s_{\chi \bar{\chi}} \leq\left(\sqrt{s}-m_{e}-m_{N}\right)^{2} .
$$

\section{APPENDIX C: SOFT-PHOTON APPROXIMATION FOR BREMSSTRAHLUNG}

Here we discuss the soft-photon approximation for bremsstrahlung, its regime of validity and explain where it fails in calculating the $2 \rightarrow 4$ cross section. In the soft limit, that is, if the emitted photon energy is small compared to the available kinetic energy $\omega \ll E_{\text {kin }}$, the 
$2 \rightarrow 3$ cross section can be factorized into an elastic scattering and an emission part, ${ }^{7}$

$d \sigma_{2 \rightarrow 3}^{\mathrm{soft}}=d \sigma_{2 \rightarrow 2} \int \frac{d^{3} k}{(2 \pi)^{3} 2 \omega} 4 \pi \alpha\left|\frac{p_{3} \cdot \epsilon^{*}}{p_{3} \cdot k}-\frac{p_{1} \cdot \epsilon^{*}}{p_{1} \cdot k}\right|^{2}$,

where $\omega^{2}=|\vec{k}|^{2}+s_{\chi \bar{\chi}}$ and $\epsilon_{\mu}$ is the polarization vector of the emitted photon. In this approximation, a simple form of the differential $2 \rightarrow 3$ cross section can be obtained in the nonrelativistic and ultrarelativistic limit respectively [85],

$$
\omega \frac{d \sigma_{2 \rightarrow 3}^{\mathrm{soft}}}{d \omega}=\left\{\begin{array}{l}
\frac{16}{3} \frac{\alpha^{3}}{\mu^{2} v^{2}} \ln \left[\frac{1+\sqrt{1-\omega / E_{\mathrm{kin}}}}{1-\sqrt{1-\omega / E_{\mathrm{kin}}}}\right] \\
\frac{4 \alpha^{3}}{\mu^{2}} \frac{E^{\prime}}{E}\left(\frac{E}{E^{\prime}}+\frac{E^{\prime}}{E}-\frac{2}{3}\right)\left(\ln \frac{E^{2} E^{\prime}}{\mu^{2} \omega}-\frac{1}{2}\right),
\end{array}\right.
$$

where $\mu$ is the reduced mass, $v$ is the relative velocity and $E\left(E^{\prime}\right)$ is the initial (final) total CM energy of the colliding particles. Even though in deriving the expression in Eq. (C2) we have assumed $\sqrt{s_{\chi \bar{\chi}}} \ll E_{\mathrm{kin}}$ and $\omega \ll E_{\mathrm{kin}}$, integrating $(\mathrm{C} 2)$ over $\omega$ in the region,

$$
\sqrt{s_{\chi \bar{\chi}}}<\omega<E_{\mathrm{kin}}
$$

still gives a very good approximation to the full cross section. Obviously, the approximation breaks down if $\sqrt{s_{\chi \bar{\chi}}} \sim E_{\mathrm{kin}}$ where the integration region of $\omega$ gets very small and the integral is dominated by large emission energies.

To obtain the $2 \rightarrow 4$ cross section from the $2 \rightarrow 3$ cross section in Eq. (24), $\sigma_{2 \rightarrow 3}^{\text {soft }}$ gets multiplied by the factors in Eq. (14) corresponding to the EM form factor interactions. This leads to the following parametric dependence on $s_{\chi \bar{\chi}}$ :

$$
d \sigma_{2 \rightarrow 4}^{\text {soft }} \propto \begin{cases}d \sigma_{2 \rightarrow 3}^{\text {soft }} d s_{\chi \bar{\chi}} / s_{\chi \bar{\chi}} & (\operatorname{dim}-4), \\ d \sigma_{2 \rightarrow 3}^{\text {soft }} d s_{\chi \bar{\chi}} & (\operatorname{dim}-5), \\ d \sigma_{2 \rightarrow 3}^{\text {soft }} d s_{\chi \bar{\chi}} s_{\chi \bar{\chi}} & (\operatorname{dim}-6),\end{cases}
$$

that is, for dim- 4 operators, like millicharged states, the $2 \rightarrow 4$ cross section is dominated by small $s_{\chi \bar{x}}$, whereas for higher dimensional operators, the expression is UV biased. Hence, the main contribution to the integral comes from $s_{\chi \bar{\chi}}$-values for which the soft approximation breaks down as one probes the kinematic end point region. It turns out that

\footnotetext{
${ }^{7}$ The emission part here describes the emission off one of the particles. If both particles can emit photons, the emission part has to be adjusted correspondingly.
}

in the nonrelativistic regime and for $m_{\chi}+m_{\bar{\chi}} \ll E_{\text {kin }}$, using Eq. (C2) reproduces the exact $2 \rightarrow 4$ cross section up to a factor 2 or 3. However, for relativistic particles, the error at $\sqrt{s_{\chi \bar{\chi}}} \sim E_{\text {kin }}$ gets larger. Due to the $s_{\chi \bar{\chi}}$-dependence in Eq. (C4), this still results in a decent description of millicharged $\chi \bar{\chi}$ emission, but produces errors of several orders of magnitude in the relativistic regime for the EM form factors considered in this paper.

Equation $(\mathrm{C} 1)$ can be further simplified by separating the phase space. This is possible, if the elastic scattering cross section is insensitive to an angular cutoff in the forward or backward direction, e.g., if the interaction is mediated by a massive particle such as the pion in $n p$ scattering. ${ }^{8}$ Then,

$$
\sigma_{2 \rightarrow 3}^{\text {soft }}=\sigma_{2 \rightarrow 2}^{\mathrm{T}} \mathcal{I}\left(s_{\chi \bar{\chi}}\right),
$$

where $\sigma_{2 \rightarrow 2}^{\mathrm{T}}$ is the transport cross section,

$$
\sigma_{2 \rightarrow 2}^{\mathrm{T}}=\int_{-1}^{1} d \cos \theta \frac{d \sigma_{2 \rightarrow 2}}{d \cos \theta}(1-\cos \theta),
$$

and the emission piece $\mathcal{I}\left(s_{\chi \bar{\chi}}\right)$ is obtained by executing the integral in Eq. (C1),

$$
\begin{aligned}
\mathcal{I}\left(s_{\chi \bar{\chi}}\right) & =\frac{1}{1-\cos \theta} \int \frac{d^{3} k}{(2 \pi)^{3} 2 \omega} 4 \pi \alpha\left|\frac{p_{3} \cdot \epsilon^{*}}{p_{3} \cdot k}-\frac{p_{1} \cdot \epsilon^{*}}{p_{1} \cdot k}\right|^{2} \\
& =\frac{\alpha}{3 \pi} \int_{\sqrt{s_{\chi \bar{X}}}}^{E_{\mathrm{kin}}} d \omega \frac{\sqrt{\omega^{2}-s_{\chi \bar{x}}}\left(s_{\chi \bar{\chi}} / 2+\omega^{2}\right)}{\omega^{4}}
\end{aligned}
$$

In the first line, we have divided by $(1-\cos \theta)$ to cancel the $\theta$-dependent part in the emission piece, which we have absorbed into the elastic cross section. In Sec. IV F we make use of this factorization in calculating the energy loss rate for neutron-proton scattering in PNS. For that, the integral in Eq. (C7) is weighted with $\omega$ to obtain

$$
\begin{aligned}
\mathcal{I}_{\omega}\left(s_{\chi \bar{\chi}}\right) & =\frac{\alpha}{3 \pi} \int_{\sqrt{s_{\bar{\chi} \bar{x}}}}^{E_{\mathrm{kin}}} d \omega \frac{\sqrt{\omega^{2}-s_{\chi \bar{\chi}}}\left(s_{\chi \bar{\chi}} / 2+\omega^{2}\right)}{\omega^{3}} \\
& =\frac{\alpha E_{\mathrm{kin}}}{3 \pi}\left[\sqrt{1-x^{2}}\left(4-x^{2}\right)-3 x \arccos (x)\right]
\end{aligned}
$$

with $x=\sqrt{s_{x \bar{x}}} / E_{\text {kin }}$, which is in agreement with the findings of Ref. [60].

\footnotetext{
${ }^{8}$ For ep scattering, on the other hand, the phase space separation is not possible, since the elastic cross section is forward divergent. In these cases, 3-body kinematics is required.
} 
[1] R. Essig et al., in Proceedings, 2013 Community Summer Study on the Future of U.S. Particle Physics: Snowmass on the Mississippi (CSS2013): Minneapolis, MN, USA (SLAC National Accelerator Laboratory, 2013).

[2] M. Battaglieri et al., arXiv:1707.04591.

[3] G. G. Raffelt, Stars as Laboratories for Fundamental Physics (University of Chicago Press, 1996).

[4] B. Holdom, Phys. Lett. 166B, 196 (1986).

[5] H. An, M. Pospelov, and J. Pradler, Phys. Lett. B 725, 190 (2013).

[6] M. Pospelov, A. Ritz, and M. B. Voloshin, Phys. Rev. D 78, 115012 (2008).

[7] J. Redondo and M. Postma, J. Cosmol. Astropart. Phys. 02 (2009) 005.

[8] J. Redondo and G. Raffelt, J. Cosmol. Astropart. Phys. 08 (2013) 034.

[9] H. An, M. Pospelov, and J. Pradler, Phys. Rev. Lett. 111, 041302 (2013).

[10] H. An, M. Pospelov, J. Pradler, and A. Ritz, Phys. Lett. B 747, 331 (2015).

[11] S. Chaudhuri, P. W. Graham, K. Irwin, J. Mardon, S. Rajendran, and Y. Zhao, Phys. Rev. D 92, 075012 (2015).

[12] S. Dubovsky and G. Hernandez-Chifflet, J. Cosmol. Astropart. Phys. 12 (2015) 054.

[13] A. Aguilar-Arevalo et al. (DAMIC Collaboration), Phys. Rev. Lett. 118, 141803 (2017).

[14] E. Hardy and R. Lasenby, J. High Energy Phys. 02 (2017) 033.

[15] R. Essig, T. Volansky, and T.-T. Yu, Phys. Rev. D 96, 043017 (2017)

[16] M. Baryakhtar, R. Lasenby, and M. Teo, Phys. Rev. D 96, 035019 (2017).

[17] V. Cardoso, P. Pani, and T.-T. Yu, Phys. Rev. D 95, 124056 (2017).

[18] M. Baryakhtar, J. Huang, and R. Lasenby, Phys. Rev. D 98 , 035006 (2018).

[19] V. Cardoso, o. J. C. Dias, G. S. Hartnett, M. Middleton, P. Pani, and J. E. Santos, J. Cosmol. Astropart. Phys. 03 (2018) 043.

[20] A. Pierce, K. Riles, and Y. Zhao, Phys. Rev. Lett. 121, 061102 (2018).

[21] M. Pospelov and T. ter Veldhuis, Phys. Lett. B 480, 181 (2000).

[22] K. Sigurdson, M. Doran, A. Kurylov, R. R. Caldwell, and M. Kamionkowski, Phys. Rev. D 70, 083501 (2004); 73, 089903(E) (2006).

[23] C. M. Ho and R. J. Scherrer, Phys. Lett. B 722, 341 (2013).

[24] D. Schmidt, T. Schwetz, and T. Toma, Phys. Rev. D 85, 073009 (2012).

[25] J. Kopp, L. Michaels, and J. Smirnov, J. Cosmol. Astropart. Phys. 04 (2014) 022.

[26] A. Ibarra and S. Wild, J. Cosmol. Astropart. Phys. 05 (2015) 047.

[27] P. Sandick, K. Sinha, and F. Teng, J. High Energy Phys. 10 (2016) 018.

[28] B. J. Kavanagh, P. Panci, and R. Ziegler, J. High Energy Phys. 04 (2019) 089.

[29] T. Trickle, Z. Zhang, and K. M. Zurek, arXiv:1905.13744.

[30] X. Chu, J. Pradler, and L. Semmelrock, Phys. Rev. D 99, 015040 (2019).
[31] B. Aubert et al. (BABAR Collaboration), Nucl. Instrum. Methods Phys. Res., Sect. A 479, 1 (2002).

[32] D. Banerjee et al. (NA64 Collaboration), Phys. Rev. D 97, 072002 (2018).

[33] A. A. Prinz et al., Phys. Rev. Lett. 81, 1175 (1998).

[34] T. Abe et al. (Belle-II Collaboration), arXiv:1011.0352.

[35] T. Åkesson et al. (LDMX Collaboration), arXiv:1808.05219.

[36] M. Battaglieri et al. (BDX Collaboration), arXiv:1607.01390.

[37] J. Bagnasco, M. Dine, and S. D. Thomas, Phys. Lett. B 320, 99 (1994).

[38] R. Foadi, M. T. Frandsen, and F. Sannino, Phys. Rev. D 80, 037702 (2009).

[39] O. Antipin, M. Redi, A. Strumia, and E. Vigiani, J. High Energy Phys. 07 (2015) 039.

[40] S. Raby and G. West, Phys. Lett. B 194, 557 (1987).

[41] M. Pospelov and A. Ritz, Phys. Rev. D 78, 055003 (2008).

[42] S. Okawa, M. Pospelov, and A. Ritz, arXiv:1905.05219.

[43] N. Viaux, M. Catelan, P. B. Stetson, G. Raffelt, J. Redondo, A. A. R. Valcarce, and A. Weiss, Phys. Rev. Lett. 111, 231301 (2013).

[44] J. A. Frieman, S. Dimopoulos, and M. S. Turner, Phys. Rev. D 36, 2201 (1987).

[45] G. G. Raffelt and G. D. Starkman, Phys. Rev. D 40, 942 (1989).

[46] N. Grevesse and A. J. Sauval, Space Sci. Rev. 85, 161 (1998).

[47] M. Asplund, N. Grevesse, A. J. Sauval, and P. Scott, Annu. Rev. Astron. Astrophys. 47, 481 (2009).

[48] K. Blum and D. Kushnir, Astrophys. J. 828, 31 (2016).

[49] N. Bar, K. Blum, and G. D'amico, arXiv:1907.05020.

[50] H. A. Weldon, Phys. Rev. D 28, 2007 (1983).

[51] M. E. Carrington, H. Defu, and R. Kobes, Phys. Rev. D 67, 025021 (2003).

[52] J. Alam, S. Sarkar, P. Roy, T. Hatsuda, and B. Sinha, Ann. Phys. (N.Y.) 286, 159 (2000).

[53] M. L. Bellac, Thermal Field Theory, Cambridge Monographs on Mathematical Physics (Cambridge University Press, Cambridge, England, 2011).

[54] E. Braaten, Phys. Rev. Lett. 66, 1655 (1991).

[55] E. Vitagliano, J. Redondo, and G. Raffelt, J. Cosmol. Astropart. Phys. 12 (2017) 010.

[56] J. H. Chang, R. Essig, and S. D. McDermott, J. High Energy Phys. 09 (2018) 051.

[57] G. G. Raffelt, Phys. Rev. D 37, 1356 (1988).

[58] P. Gondolo and G. Gelmini, Nucl. Phys. B360, 145 (1991).

[59] J. Edsjo and P. Gondolo, Phys. Rev. D 56, 1879 (1997).

[60] E. Rrapaj and S. Reddy, Phys. Rev. C 94, 045805 (2016).

[61] D. A. Brown et al., Nucl. Data Sheets 148, 1 (2018).

[62] D. Dearborn, G. Raffelt, P. Salati, J. Silk, and A. Bouquet, Astrophys. J. 354, 568 (1990).

[63] J. N. Bahcall, A. M. Serenelli, and S. Basu, Astrophys. J. 621, L85 (2005).

[64] T. Fischer, S. Chakraborty, M. Giannotti, A. Mirizzi, A. Payez, and A. Ringwald, Phys. Rev. D 94, 085012 (2016).

[65] G. G. Raffelt, Astrophys. J. 561, 890 (2001).

[66] M. T. Keil, G. G. Raffelt, and H.-T. Janka, Astrophys. J. 590, 971 (2003).

[67] C. Giunti and A. Studenikin, Rev. Mod. Phys. 87, 531 (2015).

[68] M. Tanabashi et al. (Particle Data Group), Phys. Rev. D 98, 030001 (2018). 
[69] G. G. Raffelt, Phys. Rev. Lett. 64, 2856 (1990).

[70] N. Viaux, M. Catelan, P. B. Stetson, G. Raffelt, J. Redondo, A. A. R. Valcarce, and A. Weiss, Astron. Astrophys. 558, A12 (2013).

[71] S. Arceo-Díaz, K. P. Schröder, K. Zuber, and D. Jack, Astropart. Phys. 70, 1 (2015).

[72] M. Fukugita and S. Yazaki, Phys. Rev. D 36, 3817 (1987).

[73] G. G. Raffelt and D. S. P. Dearborn, Phys. Rev. D 37, 549 (1988).

[74] G. G. Raffelt, Phys. Rep. 320, 319 (1999).

[75] J. A. Grifols and E. Masso, Phys. Rev. D 40, 3819 (1989).

[76] G. Steigman, J. Cosmol. Astropart. Phys. 04 (2010) 029.

[77] G. Mangano and P. D. Serpico, Phys. Lett. B 701, 296 (2011).
[78] J. Hamann, S. Hannestad, G. G. Raffelt, and Y. Y. Y. Wong, J. Cosmol. Astropart. Phys. 09 (2011) 034.

[79] R. Mertig, M. Bohm, and A. Denner, Comput. Phys. Commun. 64, 345 (1991).

[80] V. Shtabovenko, R. Mertig, and F. Orellana, Comput. Phys. Commun. 207, 432 (2016).

[81] E. Braaten and D. Segel, Phys. Rev. D 48, 1478 (1993).

[82] S. J. Hardy, Astron. Astrophys. 342, 614 (1999).

[83] J. F. Donoghue and B. R. Holstein, Phys. Rev. D 28, 340 (1983); 29, 3004(E) (1984).

[84] A. Lenard, Phys. Rev. 90, 968 (1953).

[85] V. Berestetskii, E. Lifshitz, and L. Pitaevskii, Quantum Electrodynamics, Course of Theoretical Physics (Butterworth-Heinemann, United Kingdom, 2012). 